Pacific

Journal of

Mathematics

\title{
CONNECTED QUANDLES ASSOCIATED WITH POINTED ABELIAN GROUPS
}

W. Edwin Clark, Mohamed Elhamdadi, Xiang-Dong Hou, MASAhico SAITO AND TIMOTHY YeATMAN 


\title{
CONNECTED QUANDLES ASSOCIATED WITH POINTED ABELIAN GROUPS
}

\author{
W. Edwin Clark, Mohamed Elhamdadi, Xiang-Dong Hou, \\ MASAHICO SAITO AND TIMOTHY YEATMAN
}

\begin{abstract}
A quandle is a self-distributive algebraic structure that appears in quasigroup and knot theories. For each abelian group $A$ and $c \in A$, we define a quandle $G(A, c)$ on $\mathbb{Z}_{3} \times A$. These quandles are generalizations of a class of nonmedial Latin quandles defined by V. M. Galkin, so we call them Galkin quandles. Each $G(A, c)$ is connected but not Latin unless $A$ has odd order. $G(A, c)$ is nonmedial unless $3 A=0$. We classify their isomorphism classes in terms of pointed abelian groups and study their various properties. A family of symmetric connected quandles is constructed from Galkin quandles, and some aspects of knot colorings by Galkin quandles are also discussed.
\end{abstract}

\section{Introduction}

Sets with certain self-distributive operations called quandles have been studied since the 1940s in various areas. They have been studied, for example, as an algebraic system for symmetries [Takasaki 1943], as quasigroups [Galkin 1988], and in relation to modules [Nelson 2003]. The fundamental quandle was defined in a manner similar to the fundamental group [Joyce 1982; Matveev 1982], which made quandles an important tool in knot theory. Algebraic homology theories for quandles were defined [Carter et al. 2003b; Fenn et al. 1995] and developed and investigated ([Litherland and Nelson 2003; Mochizuki 2011; Niebrzydowski and Przytycki 2009; 2011; Nosaka 2011], for example), and extensions of quandles by cocycles have been studied [Andruskiewitsch and Graña 2003; Carter et al. 2003a; Eisermann 2007b] and applied to various properties of knots and knotted surfaces (see [Carter et al. 2004] and references therein).

Before algebraic theories of extensions were developed, Galkin [1988] defined a family of quandles that are extensions of the 3-element connected quandle $R_{3}$, and we call them Galkin quandles. Even though the definition of Galkin quandles is a

M. S. was supported in part by NSF grant DMS 0900671.

MSC2010: 57M25.

Keywords: quandles, pointed abelian groups, knot colorings. 
special case of a cocycle extension described in [Andruskiewitsch and Graña 2003], they have curious properties such as the explicit and simple defining formula, close connections to dihedral quandles, and the fact that they appear in the list of small connected quandles.

In this paper, we generalize Galkin's definition and define a family of quandles that are extensions of $R_{3}$, characterize their isomorphism classes, and study their properties. The definition is given in Section 3 after a brief review of necessary materials in Section 2. Isomorphism classes are characterized by pointed abelian groups in Section 4. Various algebraic properties of Galkin quandles are investigated in Section 5, and their knot colorings are studied in Section 6.

\section{Preliminaries}

In this section we briefly review some definitions and examples of quandles. More details can be found, for example, in [Andruskiewitsch and Graña 2003; Carter et al. 2004; Fenn et al. 1995].

A quandle $X$ is a set with a binary operation $(a, b) \mapsto a * b$ satisfying the following conditions.

(1) (Idempotency)

(2) (Invertibility)
For any $a \in X, a * a=a$.

For any $b, c \in X$, there is a unique $a \in X$ such that $a * b=c$.

For any $a, b, c \in X$, we have $(a * b) * c=(a * c) *(b * c)$.

A quandle homomorphism between two quandles $X, Y$ is a map $f: X \rightarrow Y$ such that $f\left(x *_{X} y\right)=f(x) *_{Y} f(y)$, where $*_{X}$ and $*_{Y}$ denote the quandle operations of $X$ and $Y$, respectively. A quandle isomorphism is a bijective quandle homomorphism, and two quandles are isomorphic if there is a quandle isomorphism between them.

Typical examples of quandles include the following.

- Any nonempty set $X$ with the operation $x * y=x$ for any $x, y \in X$ is a quandle called the trivial quandle.

- A group $X=G$ with the operation of $n$-fold conjugation, $a * b=b^{-n} a b^{n}$, is a quandle.

- Let $n$ be a positive integer. For $a, b \in \mathbb{Z}_{n}$ (integers modulo $n$ ), define

$$
a * b \equiv 2 b-a(\bmod n) .
$$

Then $*$ defines a quandle structure called the dihedral quandle $R_{n}$. This set can be identified with the set of reflections of a regular $n$-gon with conjugation as the quandle operation. 
- Any $\mathbb{Z}\left[T, T^{-1}\right]$-module $M$ is a quandle with $a * b=T a+(1-T) b$ for $a, b \in M$. This is called an Alexander quandle. An Alexander quandle is also regarded as a pair $(M, T)$, where $M$ is an abelian group and $T \in \operatorname{Aut}(M)$.

Let $X$ be a quandle. The right translation $\mathscr{R}_{a}: X \rightarrow X$ by $a \in X$ is defined by $\mathscr{R}_{a}(x)=x * a$ for $x \in X$. Similarly, the left translation $\mathscr{L}_{a}$ is defined by $\mathscr{L}_{a}(x)=a * x$. Then $\mathscr{R}_{a}$ is a permutation of $X$ by Axiom (2). The subgroup of $\operatorname{Sym}(X)$ generated by the permutations $\mathscr{R}_{a}, a \in X$, is called the inner automorphism group of $X$ and is denoted by $\operatorname{Inn}(X)$. We list some definitions of commonly known properties of quandles below.

- A quandle is connected if $\operatorname{Inn}(X)$ acts transitively on $X$.

- A Latin quandle is a quandle such that for each $a \in X$, the left translation $\mathscr{L}_{a}$ is a bijection. That is, the multiplication table of the quandle is a Latin square.

- A quandle is faithful if the mapping $a \mapsto \mathscr{R}_{a}$ is an injection from $X$ to $\operatorname{Inn}(X)$.

- A quandle $X$ is involutory, or a kei, if the right translations are involutions: $\mathscr{R}_{a}^{2}=$ id for all $a \in X$.

- The operation $\bar{*}$ on $X$ defined by $a \bar{*} b=\mathscr{R}_{b}^{-1}(a)$ is a quandle operation, and $(X, \bar{*})$ is called the dual quandle of $(X, *)$. If $(X, \bar{*})$ is isomorphic to $(X, *)$, then $(X, *)$ is called self-dual.

- A quandle $X$ is medial if $(a * b) *(c * d)=(a * c) *(b * d)$ for all $a, b, c, d \in X$. It is also called abelian. It is known and easily seen that every Alexander quandle is medial.

A coloring of an oriented knot diagram by a quandle $X$ is a map $\mathscr{b}: \mathscr{A} \rightarrow X$ from the set of $\operatorname{arcs} \mathscr{A}$ of the diagram to $X$ such that the image of the map satisfies the relation depicted in Figure 1 at each crossing. More details can be found in [Carter et al. 2004; Eisermann 2007a], for example. A coloring that assigns the same element of $X$ for all the arcs is called trivial, and otherwise nontrivial. The number of colorings of a knot diagram by a finite quandle is known to be independent of the choice of a diagram, and hence is a knot invariant. A coloring by a dihedral quandle $R_{n}$ for a positive integer $n>1$ is called an $n$-coloring. If a knot is nontrivially colored by a dihedral quandle $R_{n}$ for a positive integer $n>1$, then it is called $n$-colorable. In Figure 2, a nontrivial 3-coloring of the trefoil knot ( $3_{1}$ in a common notation in a knot table [Cha and Livingston 2011]) is indicated. This is presented

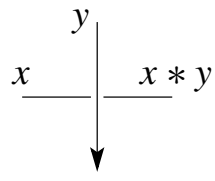

Figure 1. A coloring rule at a crossing. 


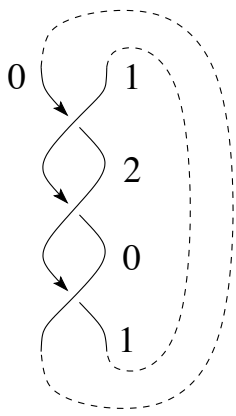

Figure 2. Trefoil as the closure of $\sigma_{1}^{3}$.

in a closed braid form. Each crossing corresponds to a standard generator $\sigma_{1}$ of the 2 -strand braid group, and $\sigma_{1}^{3}$ represents three crossings together as in the figure. The dotted line indicates the closure; see [Rolfsen 1976] for more details of braids.

The fundamental quandle is defined in a manner similar to the fundamental group [Joyce 1982; Matveev 1982]. A presentation of a quandle is defined in a manner similar to groups as well, and a presentation of the fundamental quandle is obtained from a knot diagram (see, for example, [Fenn and Rourke 1992]), by assigning generators to arcs of a knot diagram, and relations corresponding to crossings. The set of colorings of a knot diagram $K$ by a quandle $X$ is then in one-to-one correspondence with the set of quandle homomorphisms from the fundamental quandle of $K$ to $X$.

\section{Definition and notation for Galkin quandles}

Let $A$ be an abelian group, also regarded naturally as a $\mathbb{Z}$-module. Let $\mu: \mathbb{Z}_{3} \rightarrow \mathbb{Z}$, $\tau: \mathbb{Z}_{3} \rightarrow A$ be functions. These functions $\mu$ and $\tau$ need not be homomorphisms. Define a binary operation on $\mathbb{Z}_{3} \times A$ by

$$
(x, a) *(y, b)=(2 y-x,-a+\mu(x-y) b+\tau(x-y)), \quad x, y \in \mathbb{Z}_{3}, a, b \in A .
$$

Proposition 3.1. For any abelian group $A$, the operation $*$ defines a quandle structure on $\mathbb{Z}_{3} \times A$ if $\mu(0)=2, \mu(1)=\mu(2)=-1$, and $\tau(0)=0$.

Galkin [1988, p. 950] gave this definition for $A=\mathbb{Z}_{p}$. The proposition generalizes his result to any abelian group $A$. For the proof, we examine the axioms.

Lemma 3.2. (A) The operation is idempotent - that is, it satisfies Axiom (1) - if and only if $(\mu(0)-2) a=0$ for any $a \in A$, and $\tau(0)=0$.

(B) The operation as a right action is invertible - that is, it satisfies Axiom (2).

Proof. Direct calculations. 
Lemma 3.3. The operation $*$ on $\mathbb{Z}_{3} \times A$ is right self-distributive - that is, it satisfies Axiom (3) - if and only if $\mu, \tau$ satisfy the following conditions for any $X, Y \in \mathbb{Z}_{3}$ and $b, c \in A$ :

$$
\begin{aligned}
\mu(-X) b & =\mu(X) b, \\
(\mu(X+Y)+\mu(X-Y)) c & =(\mu(X) \mu(Y)) c, \\
\tau(X+Y)+\tau(Y-X) & =\tau(X)+\tau(-X)+\mu(X) \tau(Y) .
\end{aligned}
$$

Proof. Right self-distributivity, that is,

$$
((x, a) *(y, b)) *(z, c)=((x, a) *(z, c)) *((y, b) *(z, c))
$$

for $x, y, z \in \mathbb{Z}_{3}$ and $a, b, c \in A$, is satisfied if and only if

$$
\begin{aligned}
\mu(x-y) b & =\mu(y-x) b, \\
\mu(2 y-x-z) c & =(-\mu(x-z)+\mu(y-x) \mu(y-z)) c, \\
-\tau(x-y)+\tau(2 y-x-z) & =-\tau(x-z)+\mu(y-x) \tau(y-z)+\tau(y-x) .
\end{aligned}
$$

This is seen by equating the coefficients of $b$ and $c$ and the constant term. For the equivalence of the first equation with (4), set $X=x-y$. For the equivalence of the second with (5), set $X=y-x$ and $Y=z-y$. For the equivalence of the last with (6), set $X=y-x$ and $Y=y-z$.

Proof of Proposition 3.1. Assume the conditions stated. By Lemma 3.2, Axioms (1) and (2) are satisfied under the specifications $\mu(0)=2, \mu(1)=\mu(2)=-1$, and $\tau(0)=0$.

If $X=0$ or $Y=0$, then (5) (together with (4)) becomes a tautology. If $X-Y=0$ or $X+Y=0$, then (5) reduces to $\mu(2 X)+2=\mu(X)^{2}$, which is satisfied by the above specifications. For $R_{3}$, if $X+Y \neq 0$ and $X-Y \neq 0$, then either $X=0$ or $Y=0$. Hence (5) is satisfied. For (6), it is checked similarly, for the two cases $[X=0$ or $Y=0]$ and $[X-Y=0$ or $X+Y=0]$.

Definition 3.4. Let $A$ be an abelian group. The quandle defined by $*$ on $\mathbb{Z}_{3} \times A$ by Proposition 3.1,

$$
(x, a) *(y, b)=(2 y-x,-a+\mu(x-y) b+\tau(x-y)), \quad x, y \in \mathbb{Z}_{3}, a, b \in A,
$$

with $\mu(0)=2, \mu(1)=\mu(2)=-1$, and $\tau(0)=0$, is called the Galkin quandle and denoted by $G(A, \tau)$.

Since $\tau$ is specified by the values $\tau(1)=c_{1}$ and $\tau(2)=c_{2}$ where $c_{1}, c_{2} \in A$, we also denote it by $G\left(A, c_{1}, c_{2}\right)$.

Example 3.5. The Galkin quandle $G\left(\mathbb{Z}_{2}, 0,1\right)$ is $\mathbb{Z}_{3} \times \mathbb{Z}_{2}$ as a set with the quandle operation defined as above with $\mu(0)=2, \mu(1)=\mu(2)=-1, \tau(0)=\tau(1)=0$, and 
$\tau(2)=1$. Thus, $(0,1) *(1,0)=(2,-1+\mu(2) 0+\tau(2))=(2,0)$ and $(2,0) *(1,1)=$ $(0,0+\mu(1) 1+\tau(1))=(0,1)$, for example.

Lemma 3.6. For any abelian group $A$ and $c_{1}, c_{2} \in A$, the quandles $G\left(A, c_{1}, c_{2}\right)$ and $G\left(A, 0, c_{2}-c_{1}\right)$ are isomorphic.

Proof. Let $c=c_{2}-c_{1}$. Define $\eta: G\left(A, c_{1}, c_{2}\right) \rightarrow G(A, 0, c)$, as a map on $\mathbb{Z}_{3} \times A$, by $\eta(x, a)=(x, a+\beta(x))$ where $\beta(0)=\beta(1)=0$ and $\beta(2)=-c_{1}$. This $\eta$ is a bijection, and we show that it is a quandle homomorphism. We compute $\eta((x, a) *(y, b))$ and $\eta(x, a) * \eta(y, b)$ for $x, y \in \mathbb{Z}_{3}$ and $a, b \in A$.

If $x=y$, then $\mu(x-y)=2$ and $\tau(x-y)=0$ for both $G\left(A, c_{1}, c_{2}\right)$ and $G(A, 0, c)$, so that

$$
\begin{aligned}
\eta((x, a) *(x, b)) & =\eta(x, 2 b-a)=(x, 2 b-a+\beta(x)), \\
\eta(x, a) * \eta(x, b) & =(x, a+\beta(x)) *(x, b+\beta(x))=(x, 2(b+\beta(x))-(a+\beta(x))) \\
& =(x, 2 b-a+\beta(x)),
\end{aligned}
$$

as desired.

If $x-y=1 \in \mathbb{Z}_{3}$, then $\mu(x-y)=-1$ for both $G\left(A, c_{1}, c_{2}\right)$ and $G(A, 0, c)$ and $\tau(x-y)=c_{1}$ for $G\left(A, c_{1}, c_{2}\right)$ but $\tau(x-y)=0$ for $G(A, 0, c)$, so that

$$
\begin{aligned}
\eta((x, a) *(y, b)) & =\eta\left(2 y-x,-a-b+c_{1}\right)=\left(2 y-x,-a-b+c_{1}+\beta(2 y-x)\right), \\
\eta(x, a) * \eta(y, b) & =(x, a+\beta(x)) *(y, b+\beta(y)) \\
& =(2 y-x,-(a+\beta(x))-(b+\beta(y))) .
\end{aligned}
$$

The two expressions are equal if and only if $\beta(x)+\beta(y)+\beta(2 y-x)=-c_{1}$, which is true since $x \neq y$ implies that exactly one of $x, y, 2 y-x$ is $2 \in \mathbb{Z}_{3}$.

If $x-y=2 \in \mathbb{Z}_{3}$, then $\mu(x-y)=-1$ for both $G\left(A, c_{1}, c_{2}\right)$ and $G(A, 0, c)$ and $\tau(x-y)=c_{2}$ for $G\left(A, c_{1}, c_{2}\right)$ but $\tau(x-y)=c_{2}-c_{1}=c$ for $G(A, 0, c)$, so that

$$
\begin{aligned}
\eta((x, a) *(y, b)) & =\eta\left(2 y-x,-a-b+c_{2}\right)=\left(2 y-x,-a-b+c_{2}+\beta(2 y-x)\right), \\
\eta(x, a) * \eta(y, b) & =(x, a+\beta(x)) *(y, b+\beta(y)) \\
& =\left(2 y-x,-(a+\beta(x))-(b+\beta(y))+c_{2}-c_{1}\right) \\
& =\left(2 y-x,-a-b-\beta(x)-\beta(y)+\left(c_{2}-c_{1}\right)\right),
\end{aligned}
$$

and again these are equal for the same reason as above.

Notation. Since, by Lemma 3.6, any Galkin quandle is isomorphic to $G(A, 0, c)$ for an abelian group $A$ and $c \in A$, we denote $G(A, 0, c)$ by $G(A, c)$ for short.

Any finite abelian group is a product $\mathbb{Z}_{n_{1}} \times \cdots \times \mathbb{Z}_{n_{k}}$, where the positive integers $n_{j}$ satisfy $n_{j} \mid n_{j+1}$ for $j=1, \ldots, k-1$. In this case, any element $c \in A$ is written in a vector form $\left[c_{1}, \ldots, c_{k}\right]$, where $c_{j} \in \mathbb{Z}_{n_{j}}$. Then the corresponding Galkin quandle is denoted by $G\left(A,\left[c_{1}, \ldots, c_{k}\right]\right)$. 
Remark 3.7. We note that the definition of Galkin quandles induces a functor. Let $\mathbf{A} \mathbf{b}_{0}$ denote the category of pointed abelian groups; its objects are pairs $(A, c)$, where $A$ is an abelian group and $c \in A$, and its morphisms $f:(A, c) \rightarrow(B, d)$ are group homomorphisms $f: A \rightarrow B$ such that $f(c)=d$. Let $\mathbf{Q}$ be the category of quandles consisting of quandles as objects and quandle homomorphisms as morphisms.

Then the correspondence $(A, c) \stackrel{\mathscr{F}}{\mapsto} G(A, c)$ defines a functor $\mathscr{F}: \mathbf{A b}_{0} \rightarrow \mathbf{Q}$. It is easy to verify that if a morphism $f:(A, c) \rightarrow(B, d)$ is given, then the mapping $\mathscr{F}(f)(x, a)=(x, f(a))$ with $(x, a) \in G(A, c)=\mathbb{Z}_{3} \times A$ is a homomorphism from $G(A, c)$ to $G(B, d)$ and satisfies $\mathscr{F}(g f)=\mathscr{F}(g) \mathscr{F}(f)$ and $\mathscr{F}\left(\operatorname{id}_{(A, c)}\right)=\operatorname{id}_{G(A, c)}$.

Remark 3.8. A reader will wonder to what extent Definition 3.4 of a Galkin quandle can be generalized. We tried several generalizations. For example, if one attempts to replace 3 by an arbitrary prime $p$ in Definition 3.4, then Lemma 3.3 still holds. In this case for $p>3$, we prove in Lemma 5.14 that $\mu(x)=2$ for all $x \in \mathbb{Z}_{p}$, and computer experiments indicate that one almost always obtains a quandle if and only if $\tau=0$, in which case the quandle obtained is simply a product of dihedral quandles. We have also attempted to replace $-x+2 y$ by the Alexander quandle operation $t x+(1-t) y$ in both the left and right coordinates, but have neither been successful in finding interesting new quandles, nor been able to prove that no such generalizations exist. We note that if a generalization for $p>3$ exists, then any such quandles will be less dense than Galkin quandles, since multiples of 3 are more numerous than multiples of $p$ when $p>3$.

\section{Isomorphism classes}

In this section we classify isomorphism classes of Galkin quandles.

Lemma 4.1. Let $A$ be an abelian group, and let $h: A \rightarrow A^{\prime}$ be a group isomorphism. Then Galkin quandles $G(A, \tau)$ and $G\left(A^{\prime}, h \tau\right)$ are isomorphic as quandles.

Proof. Define $f: G(A, \tau) \rightarrow G\left(A^{\prime}, h \tau\right)$, as a map from $\mathbb{Z}_{3} \times A$ to $\mathbb{Z}_{3} \times A^{\prime}$, by $f(x, a)=(x, h(a))$. This $f$ is a bijection, and we show that it is a quandle homomorphism by computing $f((x, a) *(y, b))$ and $f(x, a) * f(y, b)$ for $x, y \in \mathbb{Z}_{3}$ and $a, b \in A$ :

$$
\begin{aligned}
f((x, a) *(y, b)) & =f(2 y-x,-a+\mu(x-y) b+\tau(x-y)) \\
& =(2 y-x, h(-a+\mu(x-y) b+\tau(x-y))), \\
f(x, a) * f(y, b) & =(x, h(a)) *(y, h(b)) \\
& =(2 y-x,-h(a)+\mu(x-y) h(b)+h \tau(x-y)) .
\end{aligned}
$$

The equality $f((x, a) *(y, b))=f(x, a) * f(y, b)$ follows from the facts that $h$ is a group homomorphism and $\mu(x-y)$ is an integer. 
Lemma 4.2. Let $c, d, n$ be positive integers. If $\operatorname{gcd}(c, n)=d$, then $G\left(\mathbb{Z}_{n}, c\right)$ is isomorphic to $G\left(\mathbb{Z}_{n}, d\right)$.

Proof. If $A=\mathbb{Z}_{n}$, then $\operatorname{Aut}(A)=\mathbb{Z}_{n}^{*}=$ units of $\mathbb{Z}_{n}$, and the divisors of $n$ are representatives of the orbits of $\mathbb{Z}_{n}^{*}$ acting on $\mathbb{Z}_{n}$.

Thus we may choose the divisors of $n$ for the values of $c$ for representing isomorphism classes of $G\left(\mathbb{Z}_{n}, c\right)$.

Corollary 4.3. If $A$ is a vector space (elementary p-group), then there are exactly two isomorphism classes of Galkin quandles $G(A, \tau)$.

Proof. If $A$ is a vector space containing nonzero vectors $c_{1}$ and $c_{2}$, then there is a nonsingular linear transformation $h$ of $A$ such that $h\left(c_{1}\right)=c_{2}$. That $G(A, 0)$ is not isomorphic to $G(A, c)$ if $c \neq 0$ follows from Lemma 4.5.

For distinguishing isomorphism classes, cycle structures of the right action are useful, and we use the following lemmas.

Lemma 4.4. For any abelian group $A$, the Galkin quandle $G(A, \tau)$ is connected. Proof. Recall that the operation is defined by the formula

$$
(x, a) *(y, b)=(2 y-x,-a+\mu(x-y) b+\tau(x-y)),
$$

with $\mu(0)=2, \mu(1)=\mu(2)=-1$, and $\tau(0)=0$. If $x \neq y$, then $(x, a) *(y, b)=$ $\left(2 y-x,-a-b+c_{i}\right)=(z, c)$, where $i=1$ or 2 and $x, y \in \mathbb{Z}_{3}$ and $a, b \in A$. Note that $\{x, y, 2 y-x\}=\mathbb{Z}_{3}$ if $x \neq y$. In particular, for any $(x, a)$ and $(z, c)$ with $x \neq z$, there is $(y, b)$ such that $(x, a) *(y, b)=(z, c)$.

For any $\left(x, a_{1}\right)$ and $\left(x, a_{2}\right)$ where $x \in \mathbb{Z}_{3}$ and $a_{1}, a_{2} \in A$, take $(z, c) \in \mathbb{Z}_{3} \times A$ such that $z \neq x$. Then there are $\left(y, b_{1}\right),\left(y, b_{2}\right)$ such that $x \neq y \neq z$ and $\left(x, a_{1}\right) *\left(y, b_{1}\right)=$ $(z, c)$ and $(z, c) *\left(y, b_{2}\right)=\left(x, a_{2}\right)$. Hence $G(A, \tau)$ is connected.

Lemma 4.5. The cycle structure of a right translation in $G(A, \tau)$, where $\tau(0)=$ $\tau(1)=0$ and $\tau(2)=c$, consists of 1-cycles, 2-cycles, and $2 k$-cycles, where $k$ is the order of $c$ in the group $A$.

Since isomorphic quandles have the same cycle structure of right translations, $G(A, c)$ and $G\left(A, c^{\prime}\right)$ for $c, c^{\prime} \in A$ are not isomorphic unless the orders of $c$ and $c^{\prime}$ coincide.

Proof. Let $\tau(0)=0, \tau(1)=0$, and $\tau(2)=c$. Then by Lemma 4.4, the cycle structure of each column is the same as the cycle structure of the right translation by $(0,0)$, that is, of the permutation $f(x, a)=(x, a) *(0,0)=(-x,-a+\tau(x))$.

We show that this permutation has cycles of length only 1,2 and twice the order of $c$ in $A$. Since $f(0, a)=(0,-a)$ for $a \in A, a \neq 0$, we have $f^{2}(0, a)=(0, a)$, so that $(0, a)$ generates a 2-cycle, or a 1-cycle if $2 a=0$. Now from $f(1, a)=(2,-a)$ and $f(2, a)=(1,-a+c)$ for $a \in A$, by induction it is easy to see that for $k>0$, 
$f^{2 k}(1, a)=(1, a+k c)$ and $f^{2 k}(2, a)=(2, a-k c)$. In the case of $(1, a), a \neq 0$, the cycle closes when $a+k c=a$ in $A$. The smallest $k$ for which this holds is the order of $c$, in which case the cycle is of length $2 k$. A cycle beginning at $(2, a)$ similarly has this same length.

Proposition 4.6. Let $n$ be a positive integer. Let $A=\mathbb{Z}_{n}$ and $c_{i}, c_{i}^{\prime} \in \mathbb{Z}_{n}$ for $i=1,2$. Two Galkin quandles $G\left(A, c_{1}, c_{2}\right)$ and $G\left(A, c_{1}^{\prime}, c_{2}^{\prime}\right)$ are isomorphic if and only if $\operatorname{gcd}\left(c_{1}-c_{2}, n\right)=\operatorname{gcd}\left(c_{1}^{\prime}-c_{2}^{\prime}, n\right)$.

Proof. If $\operatorname{gcd}\left(c_{1}-c_{2}, n\right)=\operatorname{gcd}\left(c_{1}^{\prime}-c_{2}^{\prime}, n\right)$, then they are isomorphic by Lemmas 3.6 and 4.2. The cycle structures are different if $\operatorname{gcd}\left(c_{1}-c_{2}, n\right) \neq \operatorname{gcd}\left(c_{1}^{\prime}-c_{2}^{\prime}, n\right)$ by Lemma 4.5, and hence they are not isomorphic.

Remark 4.7. The cycle structure is not sufficient for noncyclic groups $A$. For example, let $A=\mathbb{Z}_{2} \times \mathbb{Z}_{4}$. Then $G(A,[1,0])$ and $G(A,[0,2])$ have the same cycle structure for right translations, with cycle lengths $\{2,2,4,4,4,4\}$ in a multiset notation, yet they are known not to be isomorphic. (In the notation of Example 4.12 below, $G(A,[1,0])=C[24,29]$ and $G(A,[0,2])=C[24,31]$.) We note that there is no automorphism of $A$ carrying $[1,0]$ to $[0,2]$.

More generally, the isomorphism classes of Galkin quandles are characterized as follows.

Theorem 4.8. Suppose $A, A^{\prime}$ are finite abelian groups. Two Galkin quandles $G(A, \tau)$ and $G\left(A^{\prime}, \tau^{\prime}\right)$ are isomorphic if and only if there exists a group isomorphism $h: A \rightarrow A^{\prime}$ such that $h \tau=\tau^{\prime}$.

One implication in the proof of Theorem 4.8 is Lemma 4.1. For the other, first we prove the following two lemmas. We will use a well known description of the automorphisms of a finite abelian group, which can be found in [Hillar and Rhea 2007; Ranum 1907].

Lemma 4.9. Let $A$ be a finite abelian $p$-group and let $f: p A \rightarrow p A$ be an automorphism. Then $f$ can be extended to an automorphism of $A$.

Proof. Let $A=\mathbb{Z}_{p^{1}}^{n_{1}} \times \cdots \times \mathbb{Z}_{p^{k}}^{n_{k}}$. Then

$$
f\left(\left[\begin{array}{c}
p x_{2} \\
\vdots \\
p x_{k}
\end{array}\right]\right)=P\left[\begin{array}{c}
p x_{2} \\
\vdots \\
p x_{k}
\end{array}\right], \quad\left[\begin{array}{c}
x_{2} \\
\vdots \\
x_{k}
\end{array}\right] \in \mathbb{Z}_{p^{2}}^{n_{2}} \times \cdots \times \mathbb{Z}_{p^{k}}^{n_{k}},
$$

where

$$
P=\left[\begin{array}{cccc}
P_{22} & P_{23} & \cdots & P_{2 k} \\
p P_{32} & P_{33} & \cdots & P_{3 k} \\
\vdots & \vdots & & \vdots \\
p^{k-2} P_{k 2} & p^{k-3} P_{k 3} & \cdots & P_{k k}
\end{array}\right]
$$


$P_{i j} \in \mathrm{M}_{n_{i} \times n_{j}}(\mathbb{Z})$, det $P_{i i} \not \equiv 0(\bmod p)$. Entries of the vectors are elements of finite groups as specified, and entries of the block matrices are integers. Define $g: A \rightarrow A$ by

$$
g\left(\left[\begin{array}{c}
x_{1} \\
x_{2} \\
\vdots \\
x_{k}
\end{array}\right]\right)=\left[\begin{array}{ll}
I & \\
& P
\end{array}\right]\left[\begin{array}{c}
x_{1} \\
x_{2} \\
\vdots \\
x_{k}
\end{array}\right], \quad\left[\begin{array}{c}
x_{1} \\
x_{2} \\
\vdots \\
x_{k}
\end{array}\right] \in \mathbb{Z}_{p^{1}}^{n_{1}} \times \mathbb{Z}_{p^{2}}^{n_{2}} \times \cdots \times \mathbb{Z}_{p^{k}}^{n_{k}}
$$

Then $g \in \operatorname{Aut}(A)$ and $\left.g\right|_{p A}=f$.

Lemma 4.10. Let $A$ be a finite abelian $p$-group and let $a, b \in A \backslash p A$. If there exists an automorphism $f: p A \rightarrow p A$ such that $f(p a)=p b$, then there exists an automorphism $g: A \rightarrow A$ such that $g(a)=b$.

Proof. Let $A=\mathbb{Z}_{p^{1}}^{n_{1}} \times \cdots \times \mathbb{Z}_{p^{k}}^{n_{k}}$ and let $f$ be defined by (7) and (8). Write

$$
a=\left[\begin{array}{c}
a_{1} \\
\vdots \\
a_{n}
\end{array}\right], \quad b=\left[\begin{array}{c}
b_{1} \\
\vdots \\
b_{n}
\end{array}\right], \quad a_{i}, b_{i} \in \mathbb{Z}_{p^{i}}^{n_{i}}
$$

Since $f(p a)=p b$, we have

$$
p\left(P\left[\begin{array}{c}
a_{2} \\
\vdots \\
a_{n}
\end{array}\right]-\left[\begin{array}{c}
b_{2} \\
\vdots \\
b_{n}
\end{array}\right]\right)=0
$$

that is,

$$
P\left[\begin{array}{c}
a_{2} \\
\vdots \\
a_{n}
\end{array}\right]-\left[\begin{array}{c}
b_{2} \\
\vdots \\
b_{n}
\end{array}\right]=\left[\begin{array}{c}
p c_{2} \\
\vdots \\
p^{k-1} c_{k}
\end{array}\right], \quad c_{i} \in \mathbb{Z}_{p^{i}}^{n_{i}}, \quad 2 \leq i \leq k
$$

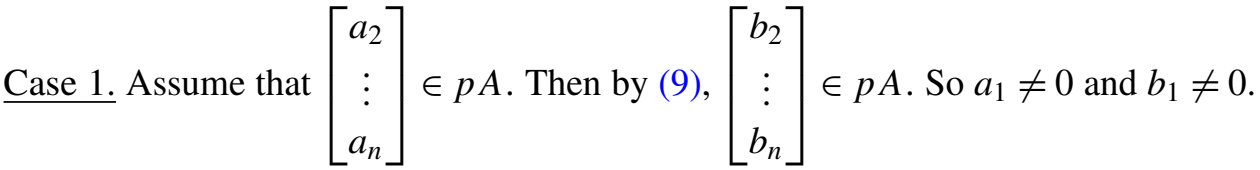
Then we have

$$
\left[\begin{array}{c}
p c_{2} \\
\vdots \\
p^{k-1} c_{k}
\end{array}\right]=\left[\begin{array}{c}
p Q_{2} \\
\vdots \\
p^{k-1} Q_{k}
\end{array}\right] a_{1}
$$

for some $Q_{i} \in \mathrm{M}_{n_{i} \times n_{1}}(\mathbb{Z})$ with $2 \leq i \leq k$. Also, there exists $P_{11} \in \mathrm{M}_{n_{1} \times n_{1}}(\mathbb{Z})$ such 
that $\operatorname{det} P_{11} \not \equiv 0(\bmod p)$ and $P_{11} a_{1}=b_{1}$. Let $g \in \operatorname{Aut}(A)$ be defined by

$$
g\left(\left[\begin{array}{c}
x_{1} \\
x_{2} \\
\vdots \\
x_{k}
\end{array}\right]\right)=\left[\begin{array}{cc}
P_{11} & 0 \\
-p Q_{2} & \\
\vdots & P \\
-p^{k-1} Q_{k} &
\end{array}\right]\left[\begin{array}{c}
x_{1} \\
x_{2} \\
\vdots \\
x_{k}
\end{array}\right], \quad x_{i} \in \mathbb{Z}_{p^{i}}^{n_{i}}
$$

Then $g(a)=b$.

Case 2. Assume that $\left[\begin{array}{c}a_{2} \\ \vdots \\ a_{n}\end{array}\right] \notin p A$. Then there exists $2 \leq s \leq k$ such that $a_{s} \notin p \mathbb{Z}_{p^{s}}^{n_{s}}$.

Then we have

$$
\left[\begin{array}{c}
c_{2} \\
\vdots \\
p^{k-2} c_{k}
\end{array}\right]=\left[\begin{array}{c}
Q_{2} \\
\vdots \\
p^{k-2} Q_{k}
\end{array}\right] a_{s}
$$

for some $Q_{i} \in \mathrm{M}_{n_{i} \times n_{s}}(\mathbb{Z})$ with $2 \leq i \leq k$. Put

$$
Q=\left[\begin{array}{ccccccc}
0 & \cdots & 0 & Q_{2} & 0 & \cdots & 0 \\
\vdots & & \vdots & \vdots & \vdots & & \vdots \\
0 & \cdots & 0 & p^{k-2} Q_{k} & 0 & \cdots & 0
\end{array}\right]
$$

where the $(i, j)$ block is of size $n_{i} \times n_{j}$ and $Q_{2}$ is in the $(1, s)$ block. Then

$$
Q\left[\begin{array}{c}
a_{2} \\
\vdots \\
a_{k}
\end{array}\right]=\left[\begin{array}{c}
c_{2} \\
\vdots \\
p^{k-2} c_{k}
\end{array}\right]
$$

Also, there exist $U \in \mathrm{M}_{n_{1} \times\left(n_{2}+\cdots+n_{k}\right)}(\mathbb{Z})$ such that

$$
U\left[\begin{array}{c}
a_{2} \\
\vdots \\
a_{k}
\end{array}\right]=b_{1}-a_{1}
$$

Now define $g \in \operatorname{Aut}(A)$ by

$$
g\left(\left[\begin{array}{c}
x_{1} \\
x_{2} \\
\vdots \\
x_{k}
\end{array}\right]\right)=\left[\begin{array}{cc}
I & U \\
0 & P-p Q
\end{array}\right]\left[\begin{array}{c}
x_{1} \\
x_{2} \\
\vdots \\
x_{k}
\end{array}\right], \quad x_{i} \in \mathbb{Z}_{p^{i}}^{n_{i}}
$$

Then $g(a)=b$. 
Proof of Theorem 4.8. We assume that $\left|3 A^{\prime}\right| \leq|3 A|$. Since $G\left(A^{\prime}, c^{\prime}\right)$ is connected, there exists an isomorphism $\phi: G(A, c) \rightarrow G\left(A^{\prime}, c^{\prime}\right)$ such that $\phi(0,0)=(0,0)$. Write

$$
\phi(x, a)=(\alpha(x, a), \beta(x, a)), \quad(x, a) \in \mathbb{Z}_{3} \times A .
$$

Define $t: \mathbb{Z}_{3} \rightarrow \mathbb{Z}$ by

$$
t(x)= \begin{cases}1 & \text { if } x=2 \\ 0 & \text { if } x \neq 2,\end{cases}
$$

so that for $(x, a),(y, b) \in \mathbb{Z}_{3} \times A$, the operation on $G(A, c)$ is written by

$$
(x, a) *(y, b)=(-x-y,-a+\mu(x-y) b+t(x-y) c) .
$$

Then $\phi((x, a) *(y, b))=\phi(x, a) * \phi(y, b)$ is equivalent to

$$
\begin{aligned}
& \alpha(-x-y,-a+\mu(x-y) b+t(x-y) c)=-\alpha(x, a)-\alpha(y, b), \\
& \beta(-x-y,-a+\mu(x-y) b+t(x-y) c) \\
& \quad=-\beta(x, a)+\mu(\alpha(x, a)-\alpha(y, b)) \beta(y, b)+t(\alpha(x, a)-\alpha(y, b)) c^{\prime} .
\end{aligned}
$$

Claim 1. The map $\alpha(0, \cdot): A \rightarrow \mathbb{Z}_{3}$ is a homomorphism.

Proof. Setting $x=y=0$ in (10), we have

$$
\alpha(0,-a+2 b)=-\alpha(0, a)-\alpha(0, b) .
$$

Setting $b=0$ in (12), we have

$$
\alpha(0,-a)=-\alpha(0, a) .
$$

By the symmetry of the right-hand side of (12), we also have

$$
\alpha(0,-a+2 b)=\alpha(0,-b+2 a), \quad a, b \in A .
$$

Now we have

$$
\begin{array}{rlrl}
\alpha(0, a+b) & =\alpha(0, a-b+2 b) & & \\
& =\alpha(0,-b+2(b-a)) & & (\text { by }(14)) \\
& =\alpha(0, b-2 a) & \\
& =-\alpha(0,-b)-\alpha(0,-a) & & (\text { by }(12)) \\
& =\alpha(0, a)+\alpha(0, b) & & (\text { by }(13)) .
\end{array}
$$

Claim 2. There exists $u \in \mathbb{Z}_{3}$ such that

$$
\alpha(x, a)=\alpha(0, a)+u x, \quad(x, a) \in \mathbb{Z}_{3} \times A .
$$


Proof. Setting $x=1$ and $y=0$ in (10), we have

$$
\alpha(-1,-a-b)=-\alpha(1, a)-\alpha(0, b) .
$$

Setting $b=0$ in (16) gives

$$
\alpha(-1,-a)=-\alpha(1, a) .
$$

Letting $a=0$ in (16) and using (17), we get

$$
\alpha(1, b)=\alpha(0, b)+\alpha(1,0), \quad b \in A .
$$

Equations (16) and (13) also imply that

$$
\alpha(-1,-b)=\alpha(0,-b)-\alpha(1,0), \quad b \in A .
$$

Let $u=\alpha(1,0)$. Then

$$
\alpha(x, a)=\alpha(0, a)+u x, \quad(x, a) \in \mathbb{Z}_{3} \times A .
$$

Claim 3.

$$
\alpha(0, c)=0 .
$$

Proof. Substituting (15) in (10), we get

$$
\alpha(0,-a+\mu(x-y) b+t(x-y) c)=-\alpha(0, a)-\alpha(0, b) .
$$

Setting $x-y=2$, we have $\alpha(0, c)=0$.

The rest of the proof of Theorem 4.8 is divided into two cases according to whether $u$ is zero or nonzero in (15).

Case A. Assume $u=0$ in (15).

We have $\alpha(x, a)=\alpha(0, a)$ for all $(x, a) \in \mathbb{Z}_{3} \times A$. We write $\alpha(a)$ for $\alpha(0, a)$. Then (11) becomes

$$
\begin{aligned}
& \beta(-x-y,-a+\mu(x-y) b+t(x-y) c) \\
& =-\beta(x, a)+\mu(\alpha(a-b)) \beta(y, b)+t(\alpha(a-b)) c^{\prime}
\end{aligned}
$$

Step A-1. We claim that $c=0$.

Equation (21) with $x=1, y=0, a=b=0$ yields

$$
\beta(-1,0)=-\beta(1,0),
$$

and with $x=-1, y=0, a=b=0$, it yields

$$
\beta(1, c)=-\beta(-1,0) .
$$

Thus $\beta(1, c)=\beta(1,0)$. Since $\alpha(1, c)=0=\alpha(1,0)$, we have $\phi(1, c)=\phi(1,0)$. Thus $c=0$.

Step A-2. We claim that $c^{\prime}=0$. 
The homomorphism $\alpha: A \rightarrow \mathbb{Z}_{3}$ must be onto. (Otherwise $\phi$ is not onto.) Choose $d \in A$ such that $\alpha(d)=-1$. Equation (21) with $x=y=0, a=d, b=0$ gives

$$
\beta(0,-d)=-\beta(0, d)+c^{\prime},
$$

and with $x=y=0, a=-d, b=0$, it gives

$$
\beta(0, d)=-\beta(0,-d) .
$$

Therefore $c^{\prime}=0$.

Step A-3. Now (21) becomes

$$
\beta(-x-y,-a+\mu(x-y) b)=-\beta(x, a)+\mu(\alpha(a-b)) \beta(y, b) .
$$

Setting $y=0$ and $b=0$ in (22), we have

$$
\beta(-x,-a)=-\beta(x, a) .
$$

Step $A$-4. We claim that $\beta(0, \cdot): 3 A \rightarrow A^{\prime}$ is a one-to-one homomorphism.

Note that $3 A \subset \operatorname{ker} \alpha$. Let $a, b \in 3 A$, and $x=-1, y=1$ in (22). We have

$$
\beta(0,-a-b)=-\beta(-1, a)+2 \beta(1, b) .
$$

Setting $b=0$ and $a=0$, respectively, in (24) and using (23), we have

$$
\begin{aligned}
& \beta(0,-a)=-\beta(-1, a)+2 \beta(1,0)=\beta(1,-a)+2 \beta(1,0), \\
& \beta(0,-b)=-\beta(-1,0)+2 \beta(1, b)=\beta(1,0)+2 \beta(1, b) .
\end{aligned}
$$

Setting $a=b=0$ in (24), we have

$$
3 \beta(1,0)=0
$$

Combining (24)-(27), we have

$$
\beta(0,-a-b)=\beta(0,-a)+\beta(0,-b) .
$$

If $a \in 3 A$ such that $\beta(0, a)=0$, then $\phi(0, a)=(0,0)$, so $a=0$. Thus

$$
\beta(0, \cdot): 3 A \rightarrow A^{\prime}
$$

is one-to-one.

Step $A-5$. We claim that $\beta(0,3 b) \in 3 A^{\prime}$ for all $b \in A$.

Let $x=y=0$ and $a=-b$ in (22). We have

$$
\begin{aligned}
\beta(0,3 b) & =-\beta(0,-b)+\mu(\alpha(-2 b)) \beta(0, b) \\
& =\beta(0, b)+\mu(\alpha(b)) \beta(0, b) \\
& \equiv 0\left(\bmod 3 A^{\prime}\right) \quad(\text { since } \mu(\alpha(b)) \equiv-1(\bmod 3)) .
\end{aligned}
$$


Step $A$-6. Now $\beta(0, \cdot): 3 A \rightarrow 3 A^{\prime}$ is a one-to-one homomorphism. It is therefore an isomorphism, since $\left|3 A^{\prime}\right| \leq|3 A|$. Since $|A|=\left|A^{\prime}\right|$, we have $A \cong A^{\prime}$. We are done in Case A.

Case B. Assume $u \neq 0$ in (15).

By the proofs of Lemma 4.5 above and Proposition 5.11 below, the map $\left(x^{\prime}, a^{\prime}\right) \mapsto$ $\left(-x^{\prime}, a^{\prime}-t\left(-x^{\prime}\right) c^{\prime}\right)$ is an isomorphism from $G\left(A^{\prime}, c^{\prime}\right)$ to $G\left(A^{\prime},-c^{\prime}\right)$. Thus we may assume $u=1$ in (15). We have $\alpha(x, a)=\alpha(0, a)+x$ for all $(x, a) \in \mathbb{Z}_{3} \times A$.

Step $B$ - 1 . We claim that $\beta(0, \cdot): \operatorname{ker} \alpha(0, \cdot) \rightarrow A^{\prime}$ is a one-to-one homomorphism. In (11) let $a, b \in \operatorname{ker} \alpha(0, \cdot)$ and $x=-1, y=1$. We have

$$
\beta(0,-a-b)=-\beta(-1, a)-\beta(1, b) .
$$

Equation (28) with $a=-b$ yields

$$
\beta(-1,-b)=-\beta(1, b) .
$$

So

$$
\beta(0,-a-b)=\beta(1,-a)-\beta(1, b) .
$$

Letting $b=0$ and $a=0$ in (30), respectively, we have

$$
\begin{aligned}
& \beta(0,-a)=\beta(1,-a)-\beta(1,0), \\
& \beta(0,-b)=\beta(1,0)-\beta(1, b) .
\end{aligned}
$$

Thus

$$
\begin{aligned}
\beta(0,-a)+\beta(0,-b) & =\beta(1,-a)-\beta(1, b) \\
& =\beta(0,-a-b) \quad(\text { by (30)). }
\end{aligned}
$$

If $a \in \operatorname{ker} \alpha(0, \cdot)$ such that $\beta(0, a)=0$, then $\phi(0, a)=(0,0)$, so $a=0$. Hence $\beta(0, \cdot): \operatorname{ker} \alpha(0, \cdot) \rightarrow A^{\prime}$ is one-to-one.

Step $B$-2. We claim that $\beta(0,3 a) \in 3 A^{\prime}$ for all $a \in A$.

Setting $x=y=0$ in (11), we have

$$
\begin{aligned}
\beta(0,-a+2 b) & =-\beta(0, a)+\mu(\alpha(0, a-b)) \beta(0, b)+t(\alpha(0, a-b)) c^{\prime} \\
& \equiv-\beta(0, a)-\beta(0, b)+t(\alpha(0, a-b)) c^{\prime}\left(\bmod 3 A^{\prime}\right) .
\end{aligned}
$$

By (31),

$$
\beta(0,3 a)=\beta(0,-a+2(2 a)) \equiv-\beta(0, a)-\beta(0,2 a)+t(\alpha(0,-a)) c^{\prime}\left(\bmod 3 A^{\prime}\right)
$$

and

$$
\beta(0,2 a)=\beta(0,0+2 a) \equiv-\beta(0, a)+t(\alpha(0,-a)) c^{\prime}\left(\bmod 3 A^{\prime}\right) .
$$

Thus $\beta(0,3 a) \equiv 0\left(\bmod 3 A^{\prime}\right)$. 
Step $B$-3. By the argument in Step A-6, $\beta(0, \cdot): 3 A \rightarrow 3 A^{\prime}$ is an isomorphism and $A \cong A^{\prime}$.

Step $B$-4. We claim that $\beta(0, c)=c^{\prime}$.

Equation (11) with $x=1, y=-1, a=b=0$ yields

$$
\begin{aligned}
\beta(0, c) & =-\beta(1,0)-\beta(-1,0)+c^{\prime} \\
& =c^{\prime} \quad(\text { by }(29)) .
\end{aligned}
$$

Step B-5. Now we complete the proof in Case B. Write $A=A_{1} \oplus A_{2}$ and $A^{\prime}=$ $A_{1}^{\prime} \oplus A_{2}^{\prime}$, where neither $\left|A_{1}\right|$ nor $\left|A_{1}^{\prime}\right|$ is a multiple of 3 , and $\left|A_{2}\right|$ and $\left|A_{2}^{\prime}\right|$ are powers of 3. Write $c=c_{1}+c_{2}$, where $c_{1} \in A_{1}, c_{2} \in A_{2}$. Then $c_{1} \in A_{1} \subset \operatorname{ker} \alpha(0, \cdot)$, so $c_{2}=c-c_{1} \in \operatorname{ker} \alpha(0, \cdot)$. Since $\beta(0, \cdot): \operatorname{ker} \alpha(0, \cdot) \rightarrow A^{\prime}$ is a homomorphism, we have

$$
c^{\prime}=\beta\left(0, c_{1}\right)+\beta\left(0, c_{2}\right)=c_{1}^{\prime}+c_{2}^{\prime},
$$

where $c_{1}^{\prime}=\beta\left(0, c_{1}\right) \in A_{1}^{\prime}$ and $c_{2}^{\prime}=\beta\left(0, c_{2}\right) \in A_{2}^{\prime}$. By Step B-3, $\beta(0, \cdot): A_{1} \rightarrow A_{1}^{\prime}$ is an isomorphism. So it suffices to show that there is an isomorphism $f: A_{2} \rightarrow A_{2}^{\prime}$ such that $f\left(c_{2}\right)=c_{2}^{\prime}$.

First assume $c_{2} \in 3 A_{2}$. Then $c_{2}^{\prime} \in 3 A_{2}^{\prime}$. By Lemma 4.9, the isomorphism $\beta(0, \cdot): 3 A \rightarrow 3 A^{\prime}$ can be extended to an isomorphism $f: A_{2} \rightarrow A_{2}^{\prime}$ and we are done.

Now assume that $c_{2} \in A_{2} \backslash 3 A_{2}$. We claim that $c_{2} \in A_{2}^{\prime} \backslash 3 A_{2}^{\prime}$. Assume to the contrary that $c_{2}^{\prime} \in 3 A_{2}^{\prime}$. By Step B-3, there exists $d \in A_{2}$ such that $\beta(0,3 d)=c_{2}^{\prime}=$ $\beta\left(0, c_{2}\right)$. By Step B-1, $c_{2}=3 d$, which is a contradiction.

Note that $\beta(0, \cdot): 3 A_{2} \rightarrow 3 A_{2}^{\prime}$ is an isomorphism and

$$
\begin{aligned}
\beta\left(0,3 c_{2}\right) & =3 \beta\left(0, c_{2}\right) \quad(\text { by Step B-1) } \\
& =3 c_{2}^{\prime} .
\end{aligned}
$$

By Lemma 4.10, there exists an isomorphism $f: A_{2} \rightarrow A_{2}^{\prime}$ such that $f\left(c_{2}\right)=c_{2}^{\prime}$.

Remark 4.11. The numbers of isomorphism classes of order $3 n$, from $n=1$ to $n=100$, are as follows: $1,2,2,5,2,4,2,10,5,4,2,10,2,4,4,20,2,10,2,10,4$, $4,2,20,5,4,10,10,2,8,2,36,4,4,4,25,2,4,4,20,2,8,2,10,10,4,2,40,5$, $10,4,10,2,20,4,20,4,4,2,20,2,4,10,65,4,8,2,10,4,8,2,50,2,4,10,10,4$, $8,2,40,20,4,2,20,4,4,4,20,2,20,4,10,4,4,4,72,2,10,10,25$.

In [Clark and Hou 2013] it is shown that the number $N(n)$ of isomorphism classes of Galkin quandles of order $n$ is multiplicative, that is, if $\operatorname{gcd}(n, m)=1$, then $N(n m)=N(n) N(m)$, so it suffices to find $N\left(q^{n}\right)$ for all prime powers $q^{n}$. Clark and Hou established that

$$
N\left(q^{n}\right)=\sum_{0 \leq m \leq n} p(m) p(n-m)
$$


where $p(m)$ is the number of partitions of the integer $m$. In particular, $N\left(q^{n}\right)$ is independent of the prime $q$. The sequence $n \mapsto N\left(q^{n}\right)$ appears in the On-Line Encyclopedia of Integer Sequences [Sloane 2011] as sequence A000712.

Example 4.12. In [Vendramin 2011], connected quandles are listed up to order 35. For a positive integer $n>1$, let $q(n)$ be the number of isomorphism classes of connected quandles of order $n$. For a positive integer $n>1$, if $q(n) \neq 0$, then we denote by $C[n, i]$ the $i$-th quandle of order $n$ in their list $(1<n \leq 35, i=1, \ldots, q(n))$. We note that $q(n)=0$ for $n=2,14,22,26$, and 34 (for $1<n \leq 35$ ). The quandle $C[n, i]$ is denoted by $Q_{n, i}$ in [Vendramin 2012] (and they are left-distributive in that work, so the matrix of $C[n, i]$ is the transpose of the matrix of $\left.Q_{n, i}\right)$. Isomorphism classes of Galkin quandles are identified with those in their list in Table 1.

The 4-digit numbers to the right of each row in Table 1 indicate the numbers of knots that are colored nontrivially by these Galkin quandles, out of total 2977 knots in the table [Cha and Livingston 2011] with 12 crossings or less. See Section 6 for more on this.

\section{Properties of Galkin quandles}

In this section, we investigate various properties of Galkin quandles.

Lemma 5.1. The Galkin quandle $G(A, \tau)$ is Latin if and only if $|A|$ is odd.

Proof. To show that it is Latin if $n$ is odd, first note that $R_{3}$ is Latin. Suppose that $(x, a) *(y, b)=(x, a) *\left(y^{\prime}, b^{\prime}\right)$. Then we have the equations

$$
\begin{aligned}
-x+2 y & =-x+2 y^{\prime}, \\
-a+\mu(x-y) b+\tau(x-y) & =-a+\mu\left(x-y^{\prime}\right) b^{\prime}+\tau\left(x-y^{\prime}\right) .
\end{aligned}
$$

From (32) it follows that $y=y^{\prime}$, and it follows from (33) that $\mu(x-y) b=\mu(x-y) b^{\prime}$. Now since $|A|$ is odd, the left module action of 2 on $A$ is invertible, and hence $b=b^{\prime}$. If $|A|$ is even, there is a nonzero element $b$ of order 2 , and hence $(0,0) *(0, b)=$ $(0,0) *(0,0)$, so the quandle is not Latin.

Lemma 5.2. Any Galkin quandle is faithful.

Proof. We show that if $(x, a) *(y, b)=(x, a) *\left(y^{\prime}, b^{\prime}\right)$ holds for all $(x, a)$, then $(y, b)=\left(y^{\prime}, b^{\prime}\right)$. We have $y=y^{\prime}$ immediately. From the second factor

$$
-a+\mu(x-y) b+\tau(x-y)=-a+\mu(x-y) b^{\prime}+\tau(x-y),
$$

we have $\mu(x-y) b=\mu(x-y) b^{\prime}$ for any $x$. Pick $x$ such that $x \neq y$; then we have $\mu(x-y)=-1$, and hence $b=b^{\prime}$.

Lemma 5.3. If $A^{\prime}$ is a subgroup of $A$ and $c^{\prime}$ is in $A^{\prime}$, then $G\left(A^{\prime}, c^{\prime}\right)$ is a subquandle of $G\left(A, c^{\prime}\right)$.

Proof. Immediate. 
Lemma 5.4. Any Galkin quandle $G(A, \tau)$ consists of three disjoint subquandles $\{x\} \times A$ for $x \in \mathbb{Z}_{3}$, and each is a product of dihedral quandles.

Proof. Immediate.

We note the following somewhat curious quandles from Lemma 5.4: For a positive integer $k, G\left(\mathbb{Z}_{2}^{k},[0, \ldots, 0]\right)$ is a connected quandle that is a disjoint union of three trivial subquandles of order $2^{k}$.

Lemma 5.5. The Galkin quandle $G(A, \tau)$ has $R_{3}$ as a subquandle if and only if $\tau=0$ or 3 divides $|A|$.

Proof. If $A$ is any group and $\tau=0$, then $(x, 0) *(y, 0)=(2 y-x, 0)$ for any $x, y \in \mathbb{Z}_{3}$, so that $\mathbb{Z}_{3} \times\{0\}$ is a subquandle isomorphic to $R_{3}$. If 3 divides $|A|$, then $A$ has a subgroup $B$ isomorphic to $\mathbb{Z}_{3}$. In the subquandle $\{0\} \times B$, we have $(0, a) *(0, b)=(0,-a+2 b)$ for $a, b \in B$, so that $\{0\} \times B$ is a subquandle isomorphic to $R_{3}$.

\begin{tabular}{|c|c|c|c|c|c|}
\hline $\begin{array}{c}\text { Rig } \\
\text { notation }\end{array}$ & $\begin{array}{c}\text { Galkin } \\
\text { notation }\end{array}$ & N.C. & $\begin{array}{c}\text { Rig } \\
\text { notation }\end{array}$ & $\begin{array}{l}\text { Galkin } \\
\text { notation }\end{array}$ & N.C. \\
\hline$C[6,1]$ & $G\left(\mathbb{Z}_{2},[0]\right)$ & 1084 & $C[24,28]$ & $G\left(\mathbb{Z}_{8},[4]\right)$ & 1084 \\
\hline$C[6,2]$ & $G\left(\mathbb{Z}_{2},[1]\right)$ & 1084 & $C[24,29]$ & $G\left(\mathbb{Z}_{2} \times \mathbb{Z}_{4},[1,0],[1,2]\right)$ & 1084 \\
\hline$C[9,2]$ & $G\left(\mathbb{Z}_{3},[0]\right)$ & 1084 & $C[24,30]$ & $G\left(\mathbb{Z}_{2} \times \mathbb{Z}_{4},\left[\begin{array}{ll}0, & 0\end{array}\right)\right.$ & 1084 \\
\hline$C[9,6]$ & $G\left(\mathbb{Z}_{3},[1]\right)$ & 1084 & $C[24,31]$ & $G\left(\mathbb{Z}_{2} \times \mathbb{Z}_{4},\left[\begin{array}{ll}0, & 2\end{array}\right]\right)$ & 1084 \\
\hline$C[12,5]$ & $G\left(\mathbb{Z}_{4},[2]\right)$ & 1084 & $C[24,32]$ & $G\left(\mathbb{Z}_{8},[1]\right)$ & 1051 \\
\hline$C[12,6]$ & $G\left(\mathbb{Z}_{4},[0]\right)$ & 1084 & $C[24,33]$ & $G\left(\mathbb{Z}_{2} \times \mathbb{Z}_{4},[0,1],[1,1]\right)$ & 1051 \\
\hline$C[12,7]$ & $G\left(\mathbb{Z}_{4},[1]\right)$ & 1051 & $C[24,38]$ & $G\left(\mathbb{Z}_{2} \times \mathbb{Z}_{2} \times \mathbb{Z}_{2},[0,0,1]\right)$ & 1084 \\
\hline$C[12,8]$ & $G\left(\mathbb{Z}_{2} \times \mathbb{Z}_{2},[0,0]\right)$ & 1084 & $C[24,39]$ & $G\left(\mathbb{Z}_{2} \times \mathbb{Z}_{2} \times \mathbb{Z}_{2},[0,0,0]\right)$ & 1084 \\
\hline$C[12,9]$ & $G\left(\mathbb{Z}_{2} \times \mathbb{Z}_{2},[1,0]\right)$ & 1084 & $C[27,2]$ & $G\left(\mathbb{Z}_{3} \times \mathbb{Z}_{3},\left[\begin{array}{ll}0, & 0\end{array}\right)\right.$ & 1084 \\
\hline$C[15,5]$ & $G\left(\mathbb{Z}_{5},[1]\right)$ & 1440 & $C[27,12]$ & $G\left(\mathbb{Z}_{9},[3]\right)$ & 1084 \\
\hline$C[15,6]$ & $G\left(\mathbb{Z}_{5},[0]\right)$ & 1512 & $C[27,13]$ & $G\left(\mathbb{Z}_{9},[0]\right)$ & 1084 \\
\hline$C[18,1]$ & $G\left(\mathbb{Z}_{2} \times \mathbb{Z}_{3},[0,0]\right)$ & 1084 & $C[27,23]$ & $G\left(\mathbb{Z}_{3} \times \mathbb{Z}_{3},[1,0]\right)$ & 1084 \\
\hline$C[18,4]$ & $G\left(\mathbb{Z}_{2} \times \mathbb{Z}_{3},[1,0]\right)$ & 1084 & $C[27,55]$ & $G\left(\mathbb{Z}_{9},[1]\right)$ & 1084 \\
\hline$C[18,5]$ & $G\left(\mathbb{Z}_{2} \times \mathbb{Z}_{3},[1,1]\right)$ & 1084 & $C[30,12]$ & $G\left(\mathbb{Z}_{2} \times \mathbb{Z}_{5},[0,1]\right)$ & 1440 \\
\hline$C[18,8]$ & $G\left(\mathbb{Z}_{2} \times \mathbb{Z}_{3},[0,1]\right)$ & 1084 & $C[30,13]$ & $G\left(\mathbb{Z}_{2} \times \mathbb{Z}_{5},[0,0]\right)$ & 1512 \\
\hline$C[21,7]$ & $G\left(\mathbb{Z}_{7},[1]\right)$ & 1339 & $C[30,14]$ & $G\left(\mathbb{Z}_{2} \times \mathbb{Z}_{5},[1,1]\right)$ & 1440 \\
\hline$C[21,8]$ & $G\left(\mathbb{Z}_{7},[0]\right)$ & 1386 & $C[30,15]$ & $G\left(\mathbb{Z}_{2} \times \mathbb{Z}_{5},[1,0]\right)$ & 1512 \\
\hline$C[24,26]$ & $G\left(\mathbb{Z}_{8},[2]\right)$ & 1071 & $C[33,10]$ & $G\left(\mathbb{Z}_{11},[0]\right)$ & 1260 \\
\hline$C[24,27]$ & $G\left(\mathbb{Z}_{8},[0]\right)$ & 1084 & $C[33,11]$ & $G\left(\mathbb{Z}_{11},[1]\right)$ & 1220 \\
\hline
\end{tabular}

Table 1. Galkin quandles in the Rig table [Vendramin 2011]. The columns headed N.C. show the number of knots with at most 12 crossings that can be nontrivially colored by the quandle. 
Conversely, let $S=\{(x, a),(y, b),(z, d)\}$ be a subquandle of $G(A, c)$ isomorphic to $R_{3}$. Note that the quandle operation of $R_{3}$ is commutative, and the product of any two distinct elements is equal to the third. We examine two cases.

Case 1. $x=y=z$. In this case we have

$$
\begin{aligned}
& (x, a) *(x, b)=(x,-a+2 b)=(x, d), \\
& (x, b) *(x, a)=(x,-b+2 a)=(x, d) .
\end{aligned}
$$

Hence we have $-a+2 b=-b+2 a$, so that $3(a-b)=0$. If there are no elements of order 3 in $A$, then we have $a-b=0$, and so $b=a$. This is a contradiction to the fact that $S$ contains 3 elements, so there is an element of order 3 in $A$; hence 3 divides $|A|$.

Case 2. $x, y$ and $z$ are all distinct (if two are distinct then all three are). In this case consider $S=\{(0, a),(1, b),(2, d)\}$. Now we have

$$
\begin{aligned}
& (2, d) *(0, a)=(1,-d-a+c)=(1, b), \\
& (0, a) *(2, d)=(1,-a-d)=(1, b) .
\end{aligned}
$$

Hence we have $-d-a+c=-a-d$, so that $c=0$, and we have $\tau=0$.

Lemma 5.6. The Galkin quandle $G(A, \tau)$ is left-distributive if and only if $3 A=0$, that is, every element of $A$ has order 3.

Proof. Let $\tau(1)=c_{1}, \tau(2)=c_{2}$. Let $a=(0,0), b=(0, \alpha)$ and $c=(1,0)$ for $\alpha \in A$. Then we get $a *(b * c)=\left(1, \alpha-c_{2}+c_{1}\right)$ and $(a * b) *(a * c)=\left(1,-2 \alpha-c_{2}+c_{1}\right)$. If these are equal, then $3 \alpha=0$ for any $\alpha \in A$.

Conversely, suppose that every element of $A$ has order 3 . Then we have $\mu(x) a=$ $2 a$ for any $x \in \mathbb{Z}_{3}, a \in A$. Then one computes

$$
\begin{aligned}
& (x, a) *[(y, b) *(z, c)]=(x *(y * z),-a+b+c-\tau(y-z)+\tau(x-y * z)), \\
& {[(x, a) *(y, b)] *[(x, a) *(z, c)]} \\
& \quad=((x * y) *(x * z),-a+b+c-\tau(x-y)-\tau(x-z)+\tau(x * y-x * z)) .
\end{aligned}
$$

If all $x, y, z$ are distinct, then $x-y=1$ or $x-y=2$, and $x * y=z, x * z=y$, $y * z=x$. If $x-y=1$, then $z=x+1$ and $y-z=1, x-z=2$, and one computes that $(34)=\left(-x+y+z,-a+b+c-c_{1}\right)=(35)$. If $x-y=2$, then one computes $(34)=\left(-x+y+z,-a+b+c-c_{2}\right)=(35)$. The other cases for $x, y, z$ are checked similarly.

Proposition 5.7. The Galkin quandle $G(A, \tau)$ is Alexander if and only if $3 A=0$.

Proof. If $G(A, \tau)$ is Alexander then it is left-distributive, and hence Lemma $5.6 \mathrm{im}$ plies $3 A=0$. Conversely, suppose $3 A=0$. Then $A=\mathbb{Z}_{3}^{k}$ for some positive integer $k$, and is an elementary 3-group. By Corollary 4.3, there are two isomorphism classes, 
$G\left(\mathbb{Z}_{3}^{k},[0, \ldots, 0]\right)$ and $G\left(\mathbb{Z}_{3}^{k},[0, \ldots, 0,1]\right)$. The quandle $G\left(\mathbb{Z}_{3}, 1\right)=C[9,6]$ is isomorphic to $\mathbb{Z}_{3}[t] /(t+1)^{2}$ by a direct comparison. Hence the two classes are isomorphic to the Alexander quandles $R_{3}^{k}$ and $R_{3}^{k-2} \times \mathbb{Z}_{3}[t] /(t+1)^{2}$, respectively.

Proposition 5.8. The Galkin quandle $G(A, c)$ is medial if and only if $3 A=0$.

Proof. We have seen that if $3 A=0$, then $G(A, c)$ is Alexander and hence is medial. Suppose $3 b \neq 0$ for some $b \in A$. Then consider the products

$$
\begin{aligned}
& X=((0,0) *(1, b)) *((1,0) *(0,0))=(-1, b-\tau(-1)), \\
& Y=((0,0) *(1,0)) *((1, b) *(0,0))=(-1,-\tau(-1)-2 b) .
\end{aligned}
$$

Since $3 b \neq 0$, we have $X \neq Y$ and so $G(A, c)$ is not medial.

Remark 5.9. The fact that the same condition appeared in Lemma 5.6 and Propositions 5.7 and 5.8 is explained as follows. Alexander quandles are left-distributive and medial. It is easy to check that, for a finite Alexander quandle $(M, T)$ with $T \in \operatorname{Aut}(M)$,

$(M, T)$ is connected $\Longleftrightarrow(1-T)$ is an automorphism of $M \Longleftrightarrow(M, T)$ is Latin.

It was also proved by Toyoda [1941] that a Latin quandle is Alexander if and only if it is medial. As noted by Galkin, $G\left(\mathbb{Z}_{5}, 0\right)$ and $G\left(\mathbb{Z}_{5}, 1\right)$ are the smallest nonmedial Latin quandles and hence the smallest non-Alexander Latin quandles.

We note that medial quandles are left-distributive (by idempotency). We show in Theorem 5.10 that any left-distributive connected quandle is Latin. This implies, by Toyoda's theorem, that every medial connected quandle is Alexander and Latin. The smallest Latin quandles that are not left-distributive are the Galkin quandles of order 15 .

It is known that the smallest left-distributive Latin quandle that is not Alexander is of order 81. This is due to V. D. Belousov. See, for example, [Pflugfelder 1990; Galkin 1988, Section 5].

Theorem 5.10. Every finite left-distributive connected quandle is Latin.

Proof. Let $(X, *)$ be a finite, connected, and left-distributive quandle. For each $a \in X$, let $X_{a}=\{a * x: x \in X\}$.

Step 1. We claim that $\left|X_{a}\right|=\left|X_{b}\right|$ for all $a, b \in X$. For any $a, y \in X$, we have

$$
\left|X_{a}\right|=\left|X_{a} * y\right|=|\{(a * x) * y: x \in X\}|=|\{(a * y) *(x * y): x \in X\}|=\left|X_{a * y}\right| .
$$

Since $X$ is connected, we have $\left|X_{a}\right|=\left|X_{b}\right|$ for all $a, b \in X$.

Step 2. Fix $a \in X$. If $\left|X_{a}\right|=|X|$, by Step $1, X_{b}=X$ for all $b \in X$ and we are done. So assume $\left|X_{a}\right|<|X|$. Clearly, $\left(X_{a}, *\right)$ is a left-distributive quandle. Since 
$(X, *)$ is connected and $x \mapsto a * x$ is an onto homomorphism from $(X, *)$ to $\left(X_{a}, *\right)$, $\left(X_{a}, *\right)$ is also connected. Using induction, we may assume that $\left(X_{a}, *\right)$ is Latin.

Step 3. For each $y \in Y$, we claim that $X_{a * y}=X_{a}$. In fact,

$$
\begin{aligned}
X_{a * y} & \supset(a * y) * X_{a} \\
& =X_{a} \quad\left(\text { since } X_{a} \text { is Latin }\right) .
\end{aligned}
$$

Since $\left|X_{a * y}\right|=\left|X_{a}\right|$, we must have $X_{a * y}=X_{a}$.

Step 4. Since $(X, *)$ is connected, by Step $3, X_{b}=X_{a}$ for all $b \in X$. Thus $X=\bigcup_{b \in X} X_{b}=X_{a}$, which is a contradiction.

Proposition 5.11. Any Galkin quandle is self-dual, that is, isomorphic to its dual.

Proof. The dual quandle structure of $G(A, \tau)=G\left(A, c_{1}, c_{2}\right)$ is written by

$$
(x, a) \bar{*}(y, b)=(x \bar{*} y,-a+\mu(y-x) b+\tau(y-x))
$$

for $(x, a),(y, b) \in G(A, \tau)$. Note that $\mu(x-y)=\mu(y-x)$ and $\tau(y-x)=c_{-i}$ if $\tau(x-y)=c_{i}$ for any $x, y \in X$ and $i \in \mathbb{Z}_{3}$. Hence its dual is $G\left(A, c_{2}, c_{1}\right)$. The isomorphism is $f: \mathbb{Z}_{3} \times A \rightarrow \mathbb{Z}_{3} \times A$, defined by $f(x, a)=(-x, a)$.

Corollary 5.12. A Galkin quandle $G\left(A, c_{1}, c_{2}\right)$ is involutory (kei) if and only if $c_{1}=c_{2} \in A$.

Proof. A quandle is a kei if and only if it is the same as its dual, that is, the identity map is an isomorphism between the dual quandle and itself. Hence this follows from Proposition 5.11.

A good involution [Kamada 2007; Kamada and Oshiro 2010] $\rho$ on a quandle $(X, *)$ is an involution $\rho: X \rightarrow X$ (a map with $\rho^{2}=\mathrm{id}$ ) such that $x * \rho(y)=x \bar{*} y$ and $\rho(x * y)=\rho(x) * y$ for any $x, y \in X$. A quandle with a good involution is called a symmetric quandle. A kei is a symmetric quandle with $\rho=\mathrm{id}$ (in this case $\rho$ is said to be trivial). Symmetric quandles have been used for unoriented knots and nonorientable surface-knots.

Symmetric quandles with nontrivial good involution have been hard to find. Other than computer calculations, very few constructions have been known. In [Kamada 2007; Kamada and Oshiro 2010], nontrivial good involutions were defined on dihedral quandles of even order, which are not connected. Infinitely many symmetric connected quandles were constructed in [Carter et al. 2010] as extensions of odd order dihedral quandles: For each odd $2 n+1(n \in \mathbb{Z}, n>0)$, a symmetric connected quandle of order $(2 n+1) 2^{2 n+1}$ was given that is not a kei. Here we use Galkin quandles to construct more symmetric quandles.

Proposition 5.13. For any positive integer $n$, there exists a symmetric connected quandle of order $6 n$ that is not involutory. 
Proof. We show that if an abelian group $A$ has an element $c \in A$ of order 2, then $G(A, c)$ is a symmetric quandle. Note that $G(A, c)$ is not involutory by Corollary 5.12.

Define the map $\rho: \mathbb{Z}_{3} \times A \rightarrow \mathbb{Z}_{3} \times A$ by $\rho(x, a)=(x, a+c)$, where $c \in A$ is a fixed element of order 2 and $x \in \mathbb{Z}_{3}, a \in A$. The map $\rho$ is an involution. It satisfies the required conditions, as we show below. For $x, y \in \mathbb{Z}_{3}$, we have

$$
\begin{aligned}
(x, a) * \rho(y, b) & =(x, a) *(y, b+c) \\
& =(2 y-x,-a+\mu(x-y)(b+c)+\tau(x-y)), \\
(x, a) \bar{*}(y, b) & =(2 y-x,-a+\mu(y-x) b+\tau(y-x)),
\end{aligned}
$$

where the last equality follows from the proof of Proposition 5.11. If $x=y$, then $\mu(x-y)=2=\mu(y-x)$ and $\tau(x-y)=0=\tau(y-x)$, and the above two terms are equal. If $x \neq y$, then $\mu(x-y)=-1=\mu(y-x)$, and exactly one of $\tau(x-y)$ and $\tau(y-x)$ is $c$ and the other is 0 , so that the equality holds.

Next we compute

$$
\begin{aligned}
\rho((x, a) *(y, b)) & =\rho(2 y-x,-a+\mu(x-y) b+\tau(x-y)) \\
& =(2 y-x,-a+\mu(x-y) b+\tau(x-y)+c), \\
\rho(x, a) *(y, b) & =(x, a+c) *(y, b) \\
& =(2 y-x,-a-c+\mu(x-y) b+\tau(x-y)),
\end{aligned}
$$

and these are equal.

For the equations in Lemma 3.3, we have the following for $\mathbb{Z}_{p}$.

Lemma 5.14. Let $p>3$ be a prime and let $\mu: \mathbb{Z}_{p} \rightarrow \mathbb{Z}$ be a function satisfying $\mu(0)=2$ and

$$
\mu(x+y)+\mu(x-y)=\mu(x) \mu(y)
$$

for any $x, y \in \mathbb{Z}_{p}$. Then $\mu(x)=2$ for all $x \in \mathbb{Z}_{p}$.

Proof. Let

$$
S=\sum_{x \in \mathbb{Z}_{p}} \mu(x) .
$$

Summing (36) as $y$ runs over $\mathbb{Z}_{p}$, we have $2 S=S \mu(x)$. So if $S \neq 0$, we have $\mu(x)=2$ for all $x \in \mathbb{Z}_{p}$. Hence we only need to prove that $S \neq 0$.

Assume to the contrary that $S=0$. Since $\mu(k x) \mu(x)=\mu((k+1) x)+\mu((k-1) x)$, it is easy to see by induction that

$$
\mu(x)^{k}=\frac{1}{2} \sum_{0 \leq i \leq k}\left(\begin{array}{l}
k \\
i
\end{array}\right) \mu((k-2 i) x) .
$$


(Here we also use the fact that $\mu(-x)=\mu(x)$, which follows from the fact that $\mu(x-y)=\mu(x) \mu(y)-\mu(x+y)$ is symmetric in $x$ and $y$.) In particular,

$$
\mu(x)^{2 p}=\frac{1}{2} \sum_{0 \leq i \leq 2 p}\left(\begin{array}{c}
2 p \\
i
\end{array}\right) \mu(2(p-i) x) .
$$

Since $\sum_{x \in \mathbb{Z}_{p}} \mu(x)=0$, we have

$$
\sum_{x \in \mathbb{Z}_{p}} \mu(x)^{2 p}=\left[2+\left(\begin{array}{c}
2 p \\
p
\end{array}\right)\right] p .
$$

Since $\mu(x)=\mu\left(\frac{x}{2}\right)^{2}-2$, we have $\mu(x)=-2,-1,2,7, \ldots$

Case 1 . Assume that there exists $0 \neq x \in \mathbb{Z}_{p}$ such that $\mu(x) \geq 7$. Then

$$
\left[2+\left(\begin{array}{c}
2 p \\
p
\end{array}\right)\right] p=\sum_{x \in \mathbb{Z}_{p}} \mu(x)^{2 p} \geq 7^{2 p},
$$

which is not possible.

Case 2. Assume that $\mu(x) \in\{-2,-1,2\}$ for all $x \in \mathbb{Z}_{p}$. Let $a_{i}=\left|\mu^{-1}(i)\right|$. Since $\sum_{x \in \mathbb{Z}_{p}} \mu(x)=0$ and $\sum_{x \in \mathbb{Z}_{p}} \mu(x)^{3}=0$, where the second equation follows from (37), we have

$$
\left\{\begin{array}{l}
-2 a_{-2}-a_{-1}+2 a_{2}=0 \\
-8 a_{-2}-a_{-1}+8 a_{2}=0
\end{array}\right.
$$

So $a_{-1}=0$, that is, $\mu(x)= \pm 2$ for all $x \in \mathbb{Z}_{p}$. Then

$$
\sum_{x \in \mathbb{Z}_{p}} \mu(x) \equiv 2 p \equiv 2(\bmod 4),
$$

which is a contradiction.

\section{Knot colorings by Galkin quandles}

In this section we investigate knot colorings by Galkin quandles. Recall from Lemma 5.4 that any Galkin quandle $G(A, \tau)$ consists of three disjoint subquandles $\{x\} \times A$ for $x \in \mathbb{Z}_{3}$, and each is a product of dihedral quandles. Also any Galkin quandle has $R_{3}$ as a quotient. Thus we look at relations between colorings by dihedral quandles and those by Galkin quandles. For a positive integer $n$, a knot is called $n$-colorable if its diagram is colored nontrivially by the dihedral quandle $R_{n}$.

First we present the numbers of $n$-colorable knots (for odd $n$ ) with 12 crossings or less out of 2977 knots in the knot table from [Cha and Livingston 2011], for comparison with Table 1. These are for dihedral quandles and their products that 
may be of interest and relevant for comparisons.

$$
\begin{array}{cccccc}
R_{3}: 1084, & R_{5}: 670, & R_{7}: 479, & R_{11}: 285, & R_{15}: 1512, & R_{17}: 192, \\
R_{19}: 159, & R_{21}: 1386, & R_{23}: 128, & R_{29}: 97, & R_{31}: 87, & R_{33}: 1260 .
\end{array}
$$

Remark 6.1. We note that many Rig Galkin quandles in Table 1 have the same number (1084) of nontrivially colorable knots as the number of 3-colorable knots. We make a few observations on these Galkin quandles.

By Lemma 5.5, a Galkin quandle has $R_{3}$ as a subquandle if $\tau=0$ or 3 divides $|A|$, and among Rig Galkin quandles with the number 1084, 17 of them satisfy this condition. Hence any 3-colorable knot is nontrivially colored by these Galkin quandles. The converse is not necessarily true: $G\left(\mathbb{Z}_{5}, 0\right)$ has $\tau=0$ but has the number 1512. See Corollary 6.5 for more on these quandles.

The remaining 7 Rig Galkin quandles with the number 1084 have $C[6,2]$ as a subquandle:

$$
C[12,5], C[12,9], C[24,28], C[24,29], C[24,31], C[24,38] \text {. }
$$

It was conjectured [Carter et al. 2010] that if a knot is 3-colorable, then it is nontrivially colored by $C[6,2]$ ( $\tilde{R}_{3}$ in their notation). It is also seen that any nontrivial coloring by $C[6,2]$ descends to a nontrivial 3-coloring via the surjection $C[6,2] \rightarrow R_{3}$, so if the conjecture is true, then any knot is nontrivially colored by these quandles if and only if it is 3-colorable. See also Remarks 6.6 and 6.7.

The determinant of a knot is a well known knot invariant related to $n$-colorability; see [Fox 1962; Rolfsen 1976] for example, for the definition.

Proposition 6.2. Let $K$ be a knot with a prime determinant $p>3$. Then $K$ is nontrivially colored by a finite Galkin quandle $G(A, \tau)$ if and only if $p$ divides $|A|$. Proof. By Fox's theorem [1962], for any prime $p$, a knot is $p$-colorable if and only if its determinant is divisible by $p$. Let $K$ be a knot with the determinant that is a prime $p>3$. Then $K$ is $p$-colorable and not 3-colorable.

Let $G(A, \tau)$ be any Galkin quandle and let $\mathscr{C}: \mathscr{A} \rightarrow G(A, \tau)$ be a coloring, where $\mathscr{A}$ is the set of arcs of a knot diagram of $K$. By the surjection $r: G(A, \tau) \rightarrow R_{3}$, the coloring $\mathscr{C}$ induces a coloring $r \circ \mathscr{C}: \mathscr{A} \rightarrow R_{3}$. Since $K$ is not 3-colorable, it is a trivial coloring, and therefore $\mathscr{C}(\mathscr{A}) \subset r^{-1}(x)$ for some $x \in R_{3}$. The subquandle $r^{-1}(x)$ for any $x \in R_{3}$ is an Alexander quandle $\{x\} \times A$ with the operation

$$
(x, a) *(x, b)=(x, 2 b-a),
$$

so that it is a product of dihedral quandles $\{x\} \times A=R_{q_{1}} \times \cdots \times R_{q_{k}}$ for some positive integer $k$ and prime powers $q_{j}, j=1, \ldots, k$ (Lemma 5.4). It is known that the number of colorings by a product quandle $X_{1} \times \cdots \times X_{k}$ is the product of numbers of colorings by $X_{i}$ for $i=1, \ldots, k$. It is also seen that a knot is nontrivially 
colored by $R_{p^{k}}$ for a prime $p$ if and only if it is $p$-colorable. Hence $K$ is nontrivially colored by $\{x\} \times A$ if and only if one of $q_{1}, \ldots, q_{k}$ is a power of $p$.

A 2-bridge knot is a knot that can be put into a position with two maxima and two minima with respect to some height function in space (see [Rolfsen 1976], for example, for its definition and properties).

Corollary 6.3. For any positive integer $n$ not divisible by 3 and any finite Galkin quandle $G(A, \tau)$, all 2-bridge knots with the determinant $n$ have the same number of colorings by $G(A, \tau)$.

Proof. Let $K$ be a two-bridge knot with the determinant $n=p_{1}^{m_{1}} \ldots p_{\ell}^{m_{\ell}}$ (in the prime decomposition form), where $p_{i} \neq 3$ for $i=1, \ldots, \ell$, and let $A=R_{q_{1}} \times \cdots \times R_{q_{k}}$ be the decomposition for prime powers, as a quandle. By Fox's theorem [1962], for a prime $p, K$ is $p$-colorable if and only if $p$ divides the determinant of $K$. Hence $K$ is $p_{i}$-colorable for $i=1, \ldots, \ell$ and not 3-colorable. By the proof of Proposition 6.2, the number of colorings by a Galkin quandle $G(A, \tau)$ of $K$ is determined by the number of colorings by the dihedral quandles $R_{q_{j}}$ that are factors of $A$.

The double branched cover $M_{2}(K)$ of the 3 -sphere $\mathbb{S}^{3}$ along a 2-bridge knot $K$ is a lens space ([Rolfsen 1976], for example), and its first homology group $H_{1}\left(M_{2}(K), \mathbb{Z}\right)$ is cyclic. If the determinant of $K$ is $n$, then it is isomorphic to $\mathbb{Z}_{n}$ ([Lickorish 1997], for example). It is known [Przytycki 1998] that the number of colorings by $R_{q_{j}}$ is equal to the order of the group $\left(\mathbb{Z} \oplus H_{1}\left(M_{2}(K), \mathbb{Z}\right)\right) \otimes \mathbb{Z}_{q_{j}}$, which is determined by $n$ and $q_{j}$ alone.

Example 6.4. Among knots with up to 8 crossings, the following sets of knots have the same numbers of colorings by all finite Galkin quandles from Corollary 6.3: $\left\{4_{1}, 5_{1}\right\}$ (determinant 5), $\left\{5_{2}, 7_{1}\right\}(7),\left\{6_{2}, 7_{2}\right\}(11),\left\{6_{3}, 7_{3}, 8_{1}\right\}(13),\left\{7_{5}, 8_{2}, 8_{3}\right\}$ (17), $\left\{7_{6}, 8_{4}\right\}(19),\left\{8_{6}, 8_{7}\right\}(23),\left\{8_{8}, 8_{9}\right\}(25),\left\{8_{12}, 8_{13}\right\}(29)$. See [Cha and Livingston 2011] for notations of knots in the table. This exhausts such sets of knots up to 8 crossings.

Computer calculations show that the set of knots up to 8 crossings with determinant 9 is $\left\{6_{1}, 8_{20}\right\}$, and these have different numbers of colorings by some Galkin quandles. The determinant was looked up at KnotInfo [Cha and Livingston 2011].

There are two knots ( $7_{4}$ and $8_{21}$, up to 8 crossings) with determinant 15 . They can be distinguished by the numbers of colorings by some Galkin quandles, according to computer calculations.

Corollary 6.5. Let $p$ be an odd prime. Then a knot $K$ is nontrivially colored by the Galkin quandle $G\left(\mathbb{Z}_{p}, 0\right)$ if and only if it is $3 p$-colorable.

Proof. Suppose it is $3 p$-colorable; then it is nontrivially colored by $R_{3 p}$, which is isomorphic to $R_{3} \times R_{p}$, so that it is either 3-colorable or $p$-colorable. If $K$ is 3 -colorable, then $K$ is nontrivially colored by $G\left(Z_{p} ; 0\right)$, since $G\left(Z_{p} ; 0\right)$ has $R_{3}$ as 
a subquandle by Lemma 5.5. If $K$ is $p$-colorable, then $K$ is nontrivially colored by $G\left(Z_{p} ; 0\right)$, since $G\left(Z_{p} ; 0\right)$ has $\{0\} \times R_{p}$ as a subquandle by Lemma 5.4.

Suppose that a knot $K$ is nontrivially colored by $G\left(\mathbb{Z}_{p}, 0\right)$, where $p$ is an odd prime. If $K$ is 3 -colorable, then it is $3 p$-colorable, and we are done. By the proof of Proposition 6.2, if $K$ is not 3-colorable, then $K$ is nontrivially colored by $\{x\} \times R_{p}$, where $x \in \mathbb{Z}_{3}$. Hence $K$ is $p$-colorable, and so $3 p$-colorable.

Remark 6.6. According to computer calculations, the following sets of Galkin quandles (in the numbering of Table 1) have the same numbers of colorings for all 2977 knots with 12 crossings or less. Thus we conjecture that it is the case for all knots. If a Galkin quandle does not appear in the list, then it means that it has different numbers of colorings for some knots, compared to other Galkin quandles. The numbers of colorings are distinct for distinct sets listed below as well.

$$
\begin{aligned}
& \{C[6,1], C[6,2]\},\{C[12,5], C[12,6]\},\{C[12,8], C[12,9]\}, \\
& \{C[18,1], C[18,4]\},\{C[18,5], C[18,8]\},\{C[24,27], C[24,28]\}, \\
& \{C[24,29], C[24,30], C[24,31]\},\{C[24,38], C[24,39]\}, \\
& \{C[30,12], C[30,14]\},\{C[30,13], C[30,15]\} .
\end{aligned}
$$

We wish to acknowledge the use of the programs GAP [2008], Maple15 (Magma package) [Maplesoft 2011], and Prover9 and Mace4 [McCune 2009] in our computations. Computational results are posted at [Clark and Yeatman 2011].

Remark 6.7. In contrast to the preceding remark, if we relax the requirement of coloring the same number of times, and instead consider two quandles equivalent if each colors the same knots nontrivially (among these 2977 knots), then we get the following 4 equivalence classes:

$\{C[3,1], C[6,1], C[6,2], C[9,2], C[9,6], C[12,5], C[12,6], C[12,8], C[12,9]$,

$C[18,1], C[18,4], C[18,5], C[18,8], C[24,27], C[24,28], C[24,29], C[24,30]$,

$C[24,31], C[24,38], C[24,39], C[27,2], C[27,12], C[27,13], C[27,23], C[27,55]\}$,

$\{C[12,7], C[24,32], C[24,33]\}$,

$\{C[15,5], C[30,12], C[30,14]\}$,

$\{C[15,6], C[30,13], C[30,15]\}$.

Thus we conjecture that it is the case for all knots. Of these, the first family with many elements consists of quandles with $C[3,1], C[6,1]$ or $C[6,2]$ as a subquandle. Hence, in fact, the conjecture about this family follows from the conjecture about $\{C[6,1], C[6,2]\}$ in the preceding remark.

Remark 6.8. Also in contrast to Remark 6.6, there exists a virtual knot $K$ (see, for example, [Kauffman 1999]) such that the numbers of colorings by $C[6,1]$ and 
$C[6,2]$ are distinct. A virtual knot $K$ with the following property was given in [Carter et al. 2010, Remark 4.6]: $K$ is 3-colorable, but does not have a nontrivial coloring by $C[6,2]$. Since $C[6,1]$ has $R_{3}$ as a subquandle, this virtual knot $K$ has a nontrivial coloring by $C[6,1]$. Hence the numbers of colorings by $C[6,1]$ and $C[6,2]$ are distinct for $K$. Thus we might conjecture that for any pair of nonisomorphic Galkin quandles, there is a virtual knot with different numbers of colorings.

Remark 6.9. For any finite Galkin quandle $G(A, \tau)$, there is a knot $K$ with a surjection $\pi_{Q}(K) \rightarrow G(A, \tau)$ from the fundamental quandle $\pi_{Q}(K)$. In fact, a connected sum of trefoils can be taken as $K$ as follows (see, for example, [Rolfsen 1976] for connected sum).

First we take a set of generators of $G(A, \tau)$ as follows. Let $A=\mathbb{Z}_{n_{1}} \times \cdots \times \mathbb{Z}_{n_{k}}$, where $k, n_{1}, \ldots, n_{k}$ are positive integers such that $n_{i}$ divides $n_{i+1}$ for $i=1, \ldots, k$. Let $S=\left\{\left(x, e_{i}\right) \mid x \in \mathbb{Z}_{3}, i=0, \ldots, k\right\}$, where $e_{0}=0 \in A$ and $e_{i} \in A(i=1, \ldots, k)$ is an elementary vector $[0, \ldots, 0,1,0, \ldots, 0] \in \mathbb{Z}_{n_{1}} \times \cdots \times \mathbb{Z}_{n_{k}}$ with a single 1 at the $i$-th position. Note that $R_{n}$ is generated by 0,1 as $0 * 1=2,1 * 2=3$, and inductively, $i *(i+1)=i+2$ for $i=0, \ldots, n-2$. Since $\{x\} \times A$ is isomorphic to a product of dihedral quandles for each $x \in \mathbb{Z}_{3}, S$ generates $G(A, \tau)$.

For a 2-string braid $\sigma_{1}^{3}$ whose closure is trefoil (see Figure 2), we note that if $x \neq y \in \mathbb{Z}_{3}$, then for any $a, b \in A$, the pair of colors $(x, a),(y, b) \in G(A, \tau)$ at top arcs extends to the bottom, that is, the bottom arcs receive the same pair. This can be computed directly.

For the copies of the trefoil, we assign pairs $\left[\left(0, e_{0}\right),\left(x, e_{i}\right)\right]$ as colors where $x=1,2$ and $i=0, \ldots k$, and take connected sums on the portion of the arcs with the common color $\left(0, e_{0}\right)$. Further we take pairs $\left[\left(0, e_{j}\right),\left(1, e_{0}\right)\right]$ for $j=1, \ldots, k$, for example, and take connected sums on the arcs with the common color $\left(1, e_{0}\right)$, to obtain a connected sum of trefoils with all elements of $S$ used as colors, as indicated in Figure 3. Such a coloring gives rise to a quandle homomorphism $\pi_{Q}(K) \rightarrow G(A, \tau)$ whose image contains generators $S$; hence it defines a surjective homomorphism.

\section{Acknowledgement}

Special thanks to Michael Kinyon for bringing [Galkin 1988] to our attention and pointing out the construction of nonmedial, Latin quandles on page 950 of [Galkin 1988] that we call here Galkin quandles $G\left(\mathbb{Z}_{p}, c_{1}, c_{2}\right)$. We are also grateful to Professor Kinyon for helping us with using Mace4 for colorings of knots by quandles, and for telling us about Belousov's work on distributive quasigroups. Thanks to David Stanovsky for useful discussions on these matters. We are grateful to James McCarron for his help with the Magma package in Maple 15, especially with isomorphism testing. We also thank the referees for valuable comments. 


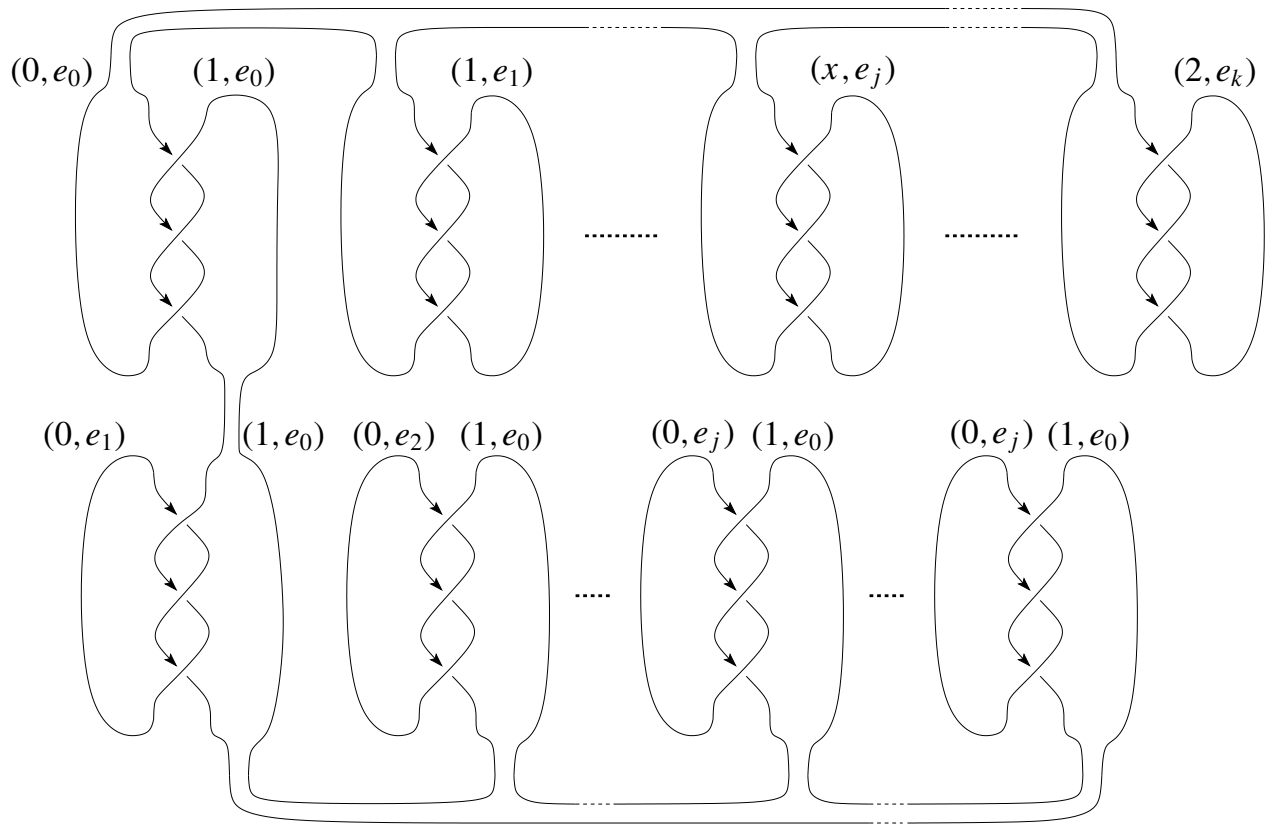

Figure 3. A coloring of a connected sum of trefoils.

\section{References}

[Andruskiewitsch and Graña 2003] N. Andruskiewitsch and M. Graña, "From racks to pointed Hopf algebras”, Adv. Math. 178:2 (2003), 177-243. MR 2004i:16046 Zbl 1032.16028

[Carter et al. 2003a] J. S. Carter, M. Elhamdadi, M. A. Nikiforou, and M. Saito, "Extensions of quandles and cocycle knot invariants", J. Knot Theory Ramifications 12:6 (2003), 725-738. MR 2004g:57020 Zbl 1049.57008

[Carter et al. 2003b] J. S. Carter, D. Jelsovsky, S. Kamada, L. Langford, and M. Saito, "Quandle cohomology and state-sum invariants of knotted curves and surfaces", Trans. Amer. Math. Soc. 355:10 (2003), 3947-3989. MR 2005b:57048 Zbl 1028.57003

[Carter et al. 2004] J. S. Carter, S. Kamada, and M. Saito, Surfaces in 4-space, Encyclopædia of Mathematical Sciences/Low-Dimensional Topology 142/III, Springer, Berlin, 2004. MR 2005e:57065 Zbl 1078.57001

[Carter et al. 2010] J. S. Carter, K. Oshiro, and M. Saito, "Symmetric extensions of dihedral quandles and triple points of non-orientable surfaces", Topology Appl. 157:5 (2010), 857-869. MR 2011c:57034 Zbl 05678844

[Cha and Livingston 2011] J. C. Cha and C. Livingston, "KnotInfo: table of knot invariants", 2011, http://www.indiana.edu/ knotinfo.

[Clark and Hou 2013] W. E. Clark and X.-D. Hou, "Galkin quandles, pointed abelian groups, and sequence A000712”, Electron. J. Combin. 20:1 (2013), P45. Zbl 06171884

[Clark and Yeatman 2011] W. E. Clark and T. Yeatman, "Small connected quandles and their knot colorings", web site, 2011, http://shell.cas.usf.edu/ saito/QuandleColor.

[Eisermann 2007a] M. Eisermann, "Knot colouring polynomials", Pacific J. Math. 231:2 (2007), 305-336. MR 2008j:57014 Zbl 1152.57010 
[Eisermann 2007b] M. Eisermann, "Quandle coverings and their Galois correspondence", preprint, 2007. arXiv math/0612459

[Fenn and Rourke 1992] R. Fenn and C. Rourke, "Racks and links in codimension two", J. Knot Theory Ramifications 1:4 (1992), 343-406. MR 94e:57006 Zbl 0787.57003

[Fenn et al. 1995] R. Fenn, C. Rourke, and B. Sanderson, "Trunks and classifying spaces", Appl. Categ. Structures 3:4 (1995), 321-356. MR 96i:57023 Zbl 0853.55021

[Fox 1962] R. H. Fox, “A quick trip through knot theory”, pp. 120-167 in Topology of 3-manifolds and related topics (Athens, GA, 1961), edited by M. K. Fort, Jr., Prentice-Hall, Englewood Cliffs, NJ, 1962. MR 25 \#3522 Zbl 06075327

[Galkin 1988] V. M. Galkin, "Quasigroups", Itogi Nauki i Tekhniki. Ser. Algebra. Topol. Geom. 26 (1988), 3-44. In Russian; translated in J. Soviet Math. 49:3 (1990), 941-967. MR 89k:20103 Zbl 0675.20057

[GAP 2008] The GAP Group, “Groups, algorithms, and programming”, Version 4.4.12, 2008, http:// www.gap-system.org.

[Hillar and Rhea 2007] C. J. Hillar and D. L. Rhea, "Automorphisms of finite abelian groups", Amer. Math. Monthly 114:10 (2007), 917-923. MR 2363058 Zbl 1156.20046

[Joyce 1982] D. Joyce, "A classifying invariant of knots, the knot quandle", J. Pure Appl. Algebra 23:1 (1982), 37-65. MR 83m:57007 Zbl 0474.57003

[Kamada 2007] S. Kamada, "Quandles with good involutions, their homologies and knot invariants", pp. 101-108 in Intelligence of low dimensional topology 2006 (Hiroshima, 2006), edited by J. S. Carter et al., Ser. Knots Everything 40, World Scientific, Hackensack, NJ, 2007. MR 2009a:57042 Zbl 0674.15021

[Kamada and Oshiro 2010] S. Kamada and K. Oshiro, "Homology groups of symmetric quandles and cocycle invariants of links and surface-links", Trans. Amer. Math. Soc. 362:10 (2010), 5501-5527. MR 2011f:57017 Zbl 1220.57016

[Kauffman 1999] L. H. Kauffman, "Virtual knot theory", European J. Combin. 20:7 (1999), 663-690. MR 2000i:57011 Zbl 0938.57006

[Lickorish 1997] W. B. R. Lickorish, An introduction to knot theory, Graduate Texts in Mathematics 175, Springer, New York, 1997. MR 98f:57015 Zbl 0886.57001

[Litherland and Nelson 2003] R. A. Litherland and S. Nelson, "The Betti numbers of some finite racks”, J. Pure Appl. Algebra 178:2 (2003), 187-202. MR 2004a:57006 Zbl 1017.55014

[Maplesoft 2011] Maplesoft, "Magma package in Maple 15”, 2011, http://www.maplesoft.com/ support/help/Maple/view.aspx?path=Magma.

[Matveev 1982] S. V. Matveev, "Distributive groupoids in knot theory", Mat. Sb. (N.S.) 119(161):1(9) (1982), 78-88. In Russian; translated in Math. USSR Sb. 47:1 (1984), 73-83. MR 84e:57008 Zbl 0523.57006

[McCune 2009] W. McCune, "Prover9 and Mace4", 2009, http://www.cs.unm.edu/ mccune/prover9.

[Mochizuki 2011] T. Mochizuki, "The third cohomology groups of dihedral quandles", J. Knot Theory Ramifications 20:7 (2011), 1041-1057. MR 2012e:18028 Zbl 1226.57010

[Nelson 2003] S. Nelson, "Classification of finite Alexander quandles", Topology Proc. 27:1 (2003), 245-258. MR 2005b:57027 Zbl 1066.57019

[Niebrzydowski and Przytycki 2009] M. Niebrzydowski and J. H. Przytycki, "Homology of dihedral quandles”, J. Pure Appl. Algebra 213:5 (2009), 742-755. MR 2010a:18014 Zbl 0302.35002

[Niebrzydowski and Przytycki 2011] M. Niebrzydowski and J. H. Przytycki, "The second quandle homology of the Takasaki quandle of an odd abelian group is an exterior square of the group", $J$. Knot Theory Ramifications 20:1 (2011), 171-177. MR 2012g:55009 Zbl 05872656 
[Nosaka 2011] T. Nosaka, "On homotopy groups of quandle spaces and the quandle homotopy invariant of links", Topology Appl. 158:8 (2011), 996-1011. MR 2012i:57024 Zbl 1227.57020

[Pflugfelder 1990] H. O. Pflugfelder, Quasigroups and loops: introduction, Sigma Series in Pure Mathematics 7, Heldermann Verlag, Berlin, 1990. MR 93g:20132 Zbl 0715.20043

[Przytycki 1998] J. H. Przytycki, "3-coloring and other elementary invariants of knots", pp. 275-295 in Knot theory (Warsaw, 1995), edited by V. F. R. Jones et al., Banach Center Publ. 42, Polish Acad. Sci., Warsaw, 1998. MR 1634462 Zbl 0904.57002 arXiv math/0608172

[Ranum 1907] A. Ranum, "The group of classes of congruent matrices with application to the group of isomorphisms of any abelian group", Trans. Amer. Math. Soc. 8:1 (1907), 71-91. MR 1500775 JFM 38.0178.01

[Rolfsen 1976] D. Rolfsen, Knots and links, Mathematics Lecture Series 7, Publish or Perish, Berkeley, CA, 1976. MR 58 \#24236 Zbl 0339.55004

[Sloane 2011] N. J. A. Sloane, "A000712: Number of permutations of $n$ into parts of 2 kinds", entry A000712 in The On-Line Encyclopedia of Integer Sequences (http://oeis.org), 2011.

[Takasaki 1943] M. Takasaki, "Abstraction of symmetric transformations", Tôhoku Math. J. 49 (1943), 145-207. In Japanese. MR 9,8c Zbl 0061.02109

[Toyoda 1941] K. Toyoda, "On axioms of linear functions", Proc. Imp. Acad. Tokyo 17:7 (1941), 221-227. MR 7,241g Zbl 0061.02403

[Vendramin 2011] L. Vendramin, "Rig: a GAP package for racks and quandles", 2011, http:// code.google.com/p/rig.

[Vendramin 2012] L. Vendramin, "On the classification of quandles of low order", J. Knot Theory Ramifications 21:9 (2012), Art. ID \#1250088. Zbl 06044378 arXiv 1105.5341

Received April 13, 2012. Revised July 13, 2012.

\section{W. EDWIN CLARK}

wclark@mail.usf.edu

Mohamed Elhamdadi

emohamed@usf.edu

XIANG-DONG HoU

xhou@usf.edu

MASAHICO SAITO

saito@usf.edu

TIMOTHY YEATMAN

tyeatma2@mail.usf.edu

(all authors)

DEPARTMENT OF MATHEMATICS AND STATistics

UNIVERSITY OF SOUTH FLORIDA

TAMPA, FL 33620-5700

UNITED STATES 


\title{
PACIFIC JOURNAL OF MATHEMATICS
}

\author{
msp.org/pjm
}

Founded in 1951 by E. F. Beckenbach (1906-1982) and F. Wolf (1904-1989)

\section{EDITORS}

V. S. Varadarajan (Managing Editor)

Department of Mathematics

University of California

Los Angeles, CA 90095-1555

pacific@math.ucla.edu

Paul Balmer

Department of Mathematics

University of California

Los Angeles, CA 90095-1555

balmer@math.ucla.edu

Daryl Cooper

Department of Mathematics

University of California

Santa Barbara, CA 93106-3080 cooper@math.ucsb.edu

Jiang-Hua $\mathrm{Lu}$

Department of Mathematics

The University of Hong Kong

Pokfulam Rd., Hong Kong jhlu@maths.hku.hk
Don Blasius

Department of Mathematics University of California

Los Angeles, CA 90095-1555

blasius@math.ucla.edu

Robert Finn

Department of Mathematics Stanford University

Stanford, CA 94305-2125

finn@math.stanford.edu

Sorin Popa

Department of Mathematics

University of California

Los Angeles, CA 90095-1555

popa@math.ucla.edu

Paul Yang

Department of Mathematics

Princeton University

Princeton NJ 08544-1000

yang@math.princeton.edu

\section{PRODUCTION}

Silvio Levy, Scientific Editor, production@msp.org

\section{SUPPORTING INSTITUTIONS}

ACADEMIA SINICA, TAIPEI

CALIFORNIA INST. OF TECHNOLOGY

INST. DE MATEMÁTICA PURA E APLICADA

KEIO UNIVERSITY

MATH. SCIENCES RESEARCH INSTITUTE

NEW MEXICO STATE UNIV.

OREGON STATE UNIV.

\author{
STANFORD UNIVERSITY \\ UNIV. OF BRITISH COLUMBIA \\ UNIV. OF CALIFORNIA, BERKELEY \\ UNIV. OF CALIFORNIA, DAVIS \\ UNIV. OF CALIFORNIA, LOS ANGELES \\ UNIV. OF CALIFORNIA, RIVERSIDE \\ UNIV. OF CALIFORNIA, SAN DIEGO \\ UNIV. OF CALIF., SANTA BARBARA
}

\author{
Vyjayanthi Chari \\ Department of Mathematics \\ University of California \\ Riverside, CA 92521-0135 \\ chari@math.ucr.edu \\ Kefeng Liu \\ Department of Mathematics \\ University of California \\ Los Angeles, CA 90095-1555 \\ liu@math.ucla.edu \\ Jie Qing \\ Department of Mathematics \\ University of California \\ Santa Cruz, CA 95064 \\ qing@cats.ucsc.edu
}

These supporting institutions contribute to the cost of publication of this Journal, but they are not owners or publishers and have no responsibility for its contents or policies.

See inside back cover or msp.org/pjm for submission instructions.

The subscription price for 2013 is US \$400/year for the electronic version, and \$485/year for print and electronic.

Subscriptions, requests for back issues and changes of subscribers address should be sent to Pacific Journal of Mathematics, P.O. Box 4163, Berkeley, CA 94704-0163, U.S.A. The Pacific Journal of Mathematics is indexed by Mathematical Reviews, Zentralblatt MATH, PASCAL CNRS Index, Referativnyi Zhurnal, Current Mathematical Publications and the Science Citation Index.

The Pacific Journal of Mathematics (ISSN 0030-8730) at the University of California, c/o Department of Mathematics, 798 Evans Hall \#3840, Berkeley, CA 94720-3840, is published monthly except July and August. Periodical rate postage paid at Berkeley, CA 94704, and additional mailing offices. POSTMASTER: send address changes to Pacific Journal of Mathematics, P.O. Box 4163, Berkeley, CA 94704-0163.

PJM peer review and production are managed by EditFLOW ${ }^{\circledR}$ from Mathematical Sciences Publishers.

PUBLISHED BY

mathematical sciences publishers

nonprofit scientific publishing

http://msp.org/

(C) 2013 Mathematical Sciences Publishers 


\section{PACIFIC JOURNAL OF MATHEMATICS}

Volume $264 \quad$ No. $1 \quad$ July 2013

On the center of fusion categories

ALAIN BRUGUIÈRES and ALEXIS VIRELIZIER

Connected quandles associated with pointed abelian groups

W. EdWin Clark, MoHamed ElHamdadi, Xiang-DONG HoU,

MASAHICO SAITO and TIMOTHY YEATMAN

Entropy and lowest eigenvalue on evolving manifolds

HongXin GuO, ROBERT PHILIPOWSKI and ANTON THALMAIER

Poles of certain residual Eisenstein series of classical groups

DIHUA JIANG, BAIYING LIU and LEI ZHANG

Harmonic maps on domains with piecewise Lipschitz continuous metrics 125

HAIGANG LI and CHANGYOU WANG

$q$-hypergeometric double sums as mock theta functions

JEREMY LOVEJOY and ROBERT OSBURN

Monic representations and Gorenstein-projective modules

XIU-HuA LUO and PU ZHANG

Helicoidal flat surfaces in hyperbolic 3-space

Antonio Martínez, JoÃo Paulo dos Santos and Keti

TENENBLAT

On a Galois connection between the subfield lattice and the multiplicative subgroup lattice

JOHN K. MCVEY

Some characterizations of Campanato spaces via commutators on Morrey 221 spaces

Shaoguang Shi and Shanzhen Lu

The Siegel-Weil formula for unitary groups 